\title{
Genome Mining of Secondary Metabolites From a Marine-derived Aspergillus Terreus B12
}

\section{xinyang du ( $\nabla 1451189146 @ q q . c o m)$}

China Pharmaceutical University College of Life Science and Technology https://orcid.org/0000-00030318-9200

\section{Huanhuan Li}

China Pharmaceutical University College of Life Science and Technology

Jiangfeng Qi

China Pharmaceutical University College of Life Science and Technology

\section{Chaoyi Chen}

China Pharmaceutical University College of Life Science and Technology

\section{Yuanyuan Lu}

China Pharmaceutical University College of Life Science and Technology

\section{Ying Wang}

China Pharmaceutical University College of Life Science and Technology https://orcid.org/0000-00025706-5361

\section{Research Article}

Keywords: Aspergillus terreus, BGCs, SMs, whole genome sequence

Posted Date: May 25th, 2021

DOI: https://doi.org/10.21203/rs.3.rs-541300/v1

License: (c) (1) This work is licensed under a Creative Commons Attribution 4.0 International License.

Read Full License 
Genome mining of secondary metabolites from a marine-derived Aspergillus terreus B12

Xinyang $\mathrm{Du}^{1}$, Huanhuan $\mathrm{Li}^{1}$, Jiangfeng Qi, Chaoyi Chen, Yuanyuan Lu, Ying Wang*

School of Life Science and Technology, China Pharmaceutical University, Nanjing 211100, China.

*Corresponding author. Tel: +86-25-86185219. fax: +86-25-8321249. E-mail: waying@.cpu.edu.cn 


\begin{abstract}
As an important saprophytic filamentous fungus, Aspergillus terreus is ubiquitously distributed, including soil rhizospheres and marine environments. Due to the prominent capabilities of bioconversion and biosynthesis, A. terreus has become attractive in biotechnical and pharmaceutical industry. In this work, an A. terreus strain, B12, was isolated from sponge in South China Sea, which demonstrated broad bacteriostatic effects against a variety of pathogenic bacteria. The whole genome was sequenced, showing a genetic richness of BGCs, which might underpin the metabolic plasticity and adaptive resilience for the strain. Genome mining identified 67 biosynthetic gene clusters (BGCs), among which, 6 gene clusters could allocate to known BGCs (100\% identity), corresponding to diverse metabolites like clavaric acid, dihydroisoflavipucine /isoflavipucine, dimethylcoprogen, alternariol, aspterric acid and pyranonigrin E. However, instead of the putative compounds, several other products were obtained from the B12 fermentation, including terrein, butyrolactone I, terretonin A\&E, acoapetaline B and epi-aszonalenins A. Of note, acoapetaline B and epi-aszonalenins A, discovered natural products recently with little information, unexpectedly were reported in this $A$. terreus strain. The genomic and heterogeneity observed in strain B12, should be at least partially attributed to the genetic variability and biochemical diversity of $A$. terreus, which could be an interesting issue open to future efforts.
\end{abstract}

Keywords: Aspergillus terreus; BGCs; SMs; whole genome sequence

Abbreviation List:

BGCs: biosynthetic gene clusters

SMs: secondary metabolites

MH: Mueller-Hinton

MEA: Malt Extract Agar

$\mathrm{MeOH}$ : Methanol

LPCB: lactophenol cotton blue

ITS: internal transcribed spacer

ML: maximum-likelihood

GO: Gene Ontology

KEGG: Kyoto Encyclopedia of Genes and Genomes

KOG: euKaryotic Orthologous Groups

CAZy: Carbohydrate-Active enZYmes Database

NRPS: nonribosomal peptide synthase

PKS: polyketide synthase

\title{
Introduction
}

As a valuable producer of secondary metabolites (SMs), marine fungi represent an 
underestimated source of biological and chemical diversity, although their distribution and ecological roles often remain scarce[1]. Owing to the particularity of marine environment, many SMs are structurally unique and possess promising biological and pharmacological properties, in comparison with their terrestrial origin counterparts[2]. For decades, despite the significant increase in the number of structures discovered from marine fungi, the species diversity and the large potential of SMs is not yet adequately represented[3].

As filter feeders in marine ecosystem, sponges form close associations with diverse groups of microbes, potentially involved in a variety of ecological functions including SMs generation, which could contribute to an ecological success, to themselves and to the host in niche competition[4, 5]. In our previous work, a range of filamentous fungal strains were isolated from sponges in the South China Sea, typically dominated by Aspergillus and Penicillium sp.[6].

Hereon, we present the draft genome of $\mathrm{B} 12$, an $A$. terreus strain with broad antibacterial activities in preliminary screening. Subsequently, genome mining, metabolites separation and structure determination were performed, partially presenting the biosynthetic potential and chemical diversity of the strain, which highlighted the need for further study and shed some lights on the ecological and biochemical properties of $A$. terreus strains.

\section{Materials and methods}

\section{conditions of fermentation}

The B12 was activated in the PDB liquid medium, and then inoculated onto corn medium (corn $100 \mathrm{~g}, \mathrm{MgSO} 40.2 \mathrm{~g}$, sea salt $1.5 \mathrm{~g}$, malt sugar $2 \mathrm{~g}$, sorbitol $2 \mathrm{~g}$; yeast extract $0.3 \mathrm{~g}$, tryptophane $0.05 \mathrm{~g}$, sodium glutamate $1 \mathrm{~g}, \mathrm{~K} 2 \mathrm{HPO} 40.05 \mathrm{~g}$, water $1 \mathrm{~L}$ ) to culture at $28{ }^{\circ} \mathrm{C}$ for 15 days. The fermented products were extracted by methanol $(\mathrm{MeOH})$ followed by decompressing distillation to acquire a crude extract. The crude extract was dissolved in $2 \mathrm{~mL} \mathrm{MeOH}$ and test for antibacterial activity by the agar diffusion method.

Escherichia coli ATCC 25922, Klebsiella aerogenes ATCC 700603, Pseudomonas aeruginosa PA101, methicillin-resistant Staphylococcus aureus (MRSA) USA300, methicillin-resistant Staphylococcus epidermidis (MRSE) ATCC 35984, Micrococcus luteus ACCC11001, and Acinetobacter baumannii ATCC 19606 were utilized as control strains. All the above strains were stored in the Marine Pharmaceutical Laboratory of China Pharmaceutical University.

single colonies of indicator bacteria was inoculated into Mueller-Hinton (MH,Solarbio, China) broth and cultured to logarithmic phase. The bacterial inoculum(OD600nn $\approx 0.1$ ) was spread onto MH agar (Solarbio, China). Wells measuring $6 \mathrm{~mm}$ in diameter were punched onto the surface of the agar using a sterile 
hole puncher. $30 \mu \mathrm{L}$ crude extract was added to the wells and incubated for $24 \mathrm{~h}$ at $28{ }^{\circ} \mathrm{C}$. Eachassessment was developed in triplicate. The standard antibiotics $(0.1 \mathrm{mM}$ chloramphenicol) were used as a positive control, $\mathrm{MeOH}$ was used as a vehicle control. The diameters (in $\mathrm{mm}$ ) of the inhibition zone were recorded to estimate antimicrobial activities, which were expressed by the ratio of the inhibition zone relative to that of the positive control. The bacteriostatic activities were considered strong if the ratio was greater than 1.0, moderate when the scale was between 0.5 and 1 , and weak if it was less than 0.5 .

\section{species identification}

The spore suspensions of B12 was inoculated and grown on Malt Extract Agar (MEA, OXOID, UK) which adding $3 \%$ sea malt, $28{ }^{\circ} \mathrm{C}$ for 5 days. The colony was observed and characterized, such as size, texture, color, soluble pigments, and exudates. Next, microscopic examination was performed on spores and hyphae followed by lactophenol cotton blue (LPCB) staining[7]under a BA210 light microscope (Motic, Xiamen, China).

Molecular markers have been widely approved as crucial for the taxonomic identification of fungi, such as ITS[8, 9]. In our work, the phylogenetic status of the B12 was analyzed based on the ITS sequences. Phylogenetic trees were constructed by dataset of ITS sequences and concatenated sequences using maximum-likelihood (ML) analysis in MEGA7.

\section{Genome sequencing}

Due to the relatively wide anti-bactericidal activity of B12 ferment, suggest that the vital role played by SMs and the generation process. In order to explore the potential of produce the SMs of B12, we sequenced the whole genome of B12.In brief, the construction of DNA libraries was performed using $100 \mathrm{ng}$ genomic DNA, which was randomly fragmented to 500 bp by sonication (Covaris S220, USA). Sequencing was subsequently performed using a $2 \times 150$-bp paired-end (PE) configuration; image analysis and base calling were performed using HiSeq Control Software. The adapter and low-quality sequences were removed from the raw sequencing data by cutadapt (v1.9.1). The ideal reads were assembled and gap-filled using Velvet[10], SSPACE[11] and GapFiller[12], respectively. All genome sequencing data have been submitted to the NCBI SRA database. The SRA accession numbers of B12 is PRJNA714189.

The genomic sequences of B12 was analyzed with the antiSMASH[13] online server with the ClusterFinder algorithm to identify the potential biosynthetic gene clusters (BGCs) based on homology analysis.

\section{Gene prediction and functional annotation}


The software Augustus (version 3.3)[14] was used to predict coding genes and high-GC regions. Through a homology-based approach, the gene structures were mapped to the reference genome Aspergillus terreus NIH2624. Next, the coding genes were annotated with the NCBI nr database by BLAST, and the functions of genes were annotated by the Gene Ontology (GO) [15] and Kyoto Encyclopedia of Genes and Genomes (KEGG)[16] databases. In addition, the predicted proteins were classified by the Cluster of Orthologous Groups of proteins (KOG) database[17].

\section{Results}

\section{Antibacterial activities screening}

A range of pathogenic bacteria were hired to assess the antimicrobial property of B12 (Fig.1). Relative to equivoluminal antibiotics (0.1 $\mathrm{mM}$ chloramphenicol), the fermented products of strain B12 displayed a considerable and broad-spectrum growth inhibition against almost all of tested bacteria. More precisely, the crude extract from B12 fermentation exhibited more potent effects against Gram-positive bacteria (MRSA, MRSE, M.luteus, S.aureus) than against Gram-negative bacteria (E.coli, A. baumannii, K.aerogenes, P.aeruginosa), specified by the bacteriostatic efficacy relative to standard antibiotics $(0.1 \mathrm{mM}$ chloramphenicol). In view of the unique ecological fitness, further genetic and physiological investigations are necessary on the strain to unveil the underlying bioactive components and possible synthetic profiles.

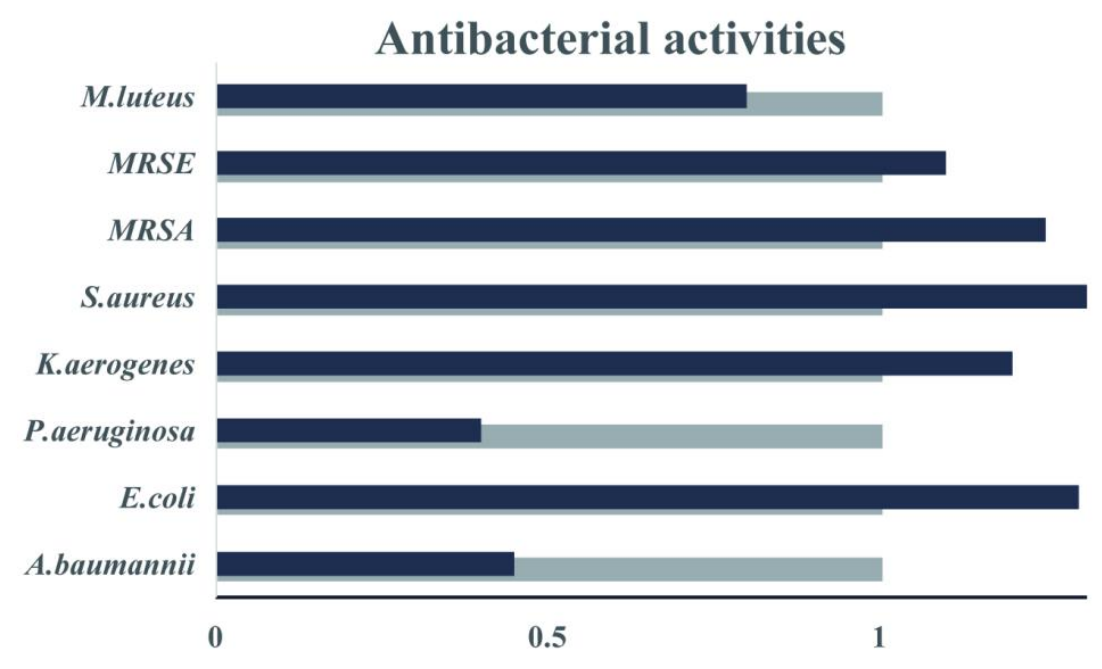

Figure 1. the antibacterial activities of the extractive of B12. The standard antibiotics $(0.1 \mathrm{mM}$ chloramphenicol) were used as a positive control, $\mathrm{MeOH}$ was used as a vehicle. The diameters (in $\mathrm{mm}$ ) of the inhibition zone were recorded to 
estimate antimicrobial activities, which were expressed by the ratio of the inhibition zone relative to that of the positive control ( $0.1 \mathrm{mM}$ chloramphenicol ) . The bacteriostatic activities were considered strong if the ratio was greater than 1.0, moderate when the scale was between 0.5 and 1 , and weak if it was less than 0.5.the blue bar indicated as 1 , the black-blue indicated as the ratio of the inhibition zone relative to that of the positive control. E. coli: Escherichia coli; P. aeruginosa: Pseudomonas aeruginosa; K. aerogenes: Klebsiella aerogenes; MRSA: methicillin-resistant Staphylococcus aureus; MRSE: methicillin-resistant Staphylococcus epidermidis; $M$. luteus: Micrococcus luteus; A. baumannii: Acinetobacter baumannii.

\section{Morphological and phylogenetic analysis}

In microbial taxonomy, classic species features such as morphological, physiological and genetic characteristics have become essential and canonical approaches. The purified fungus strain B12 was first characterized based on morphological, microscopic and molecular characteristics.

As shown by morphological features, including conidiophores, hyphae, pigments, and colony appearance, the strain grows slowly after 5 days at $25^{\circ} \mathrm{C}$ on MEA, with white, circular and flocculose colonies. The colonies are slightly convex, margin filiform, lanose; brownish orange reverse center becomes towards the grey margin pale without soluble pigment or exudate diffusing to the agar. Microscopy shows that mycelium grows filiform, while small and globose conidia stack to form compact conidial heads (Fig.2 A). Macroscopically or microscopically, the strain B12 could be identified as Aspergillus sp. by those morphological features, which certainly requires further identification by molecular techniques.

Among the regions of the ribosomal cistron, internal transcribed spacer (ITS) region provides the highest resolving power for discriminating closely related species, having been adopted as the primary barcode marker for fungi[18]. In our study, based on the ITS sequence, the phylogenetic tree was constructed among fungi, which clearly delineated species delimitation between Cladosporium and Aspergillus. Consistent with morphological characterization, B12 was clustered into Aspergillus clades, supported by the highest identity scores and $100 \%$ bootstrap value. More specifically, the strain closely associated with Aspergillus terreus, forming a sister taxon with Aspergillus niger clade (Fig.2 B). 
A
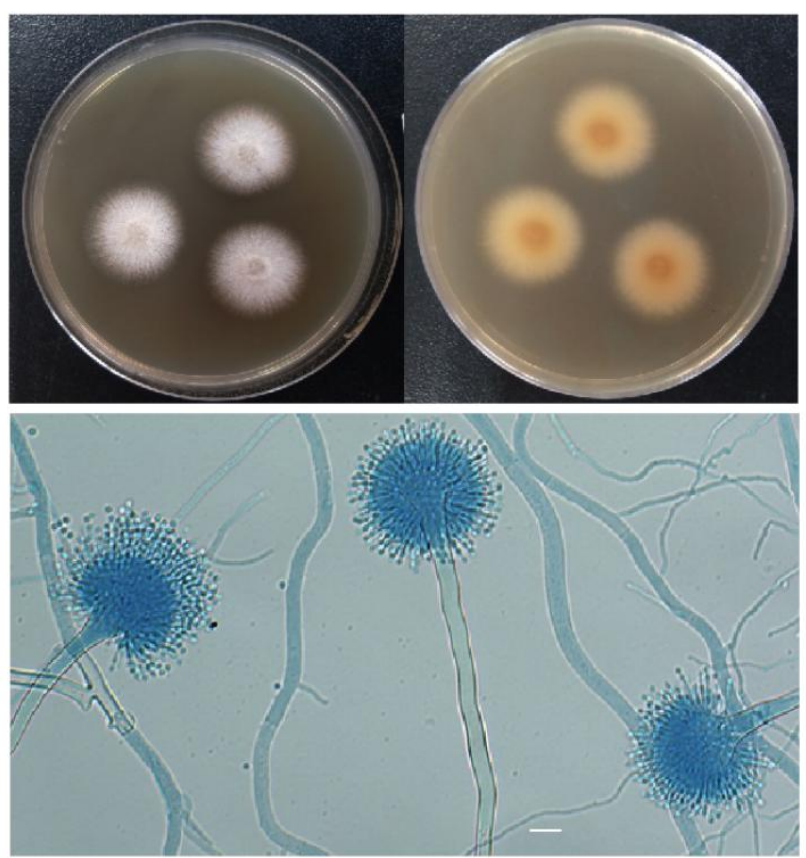

B

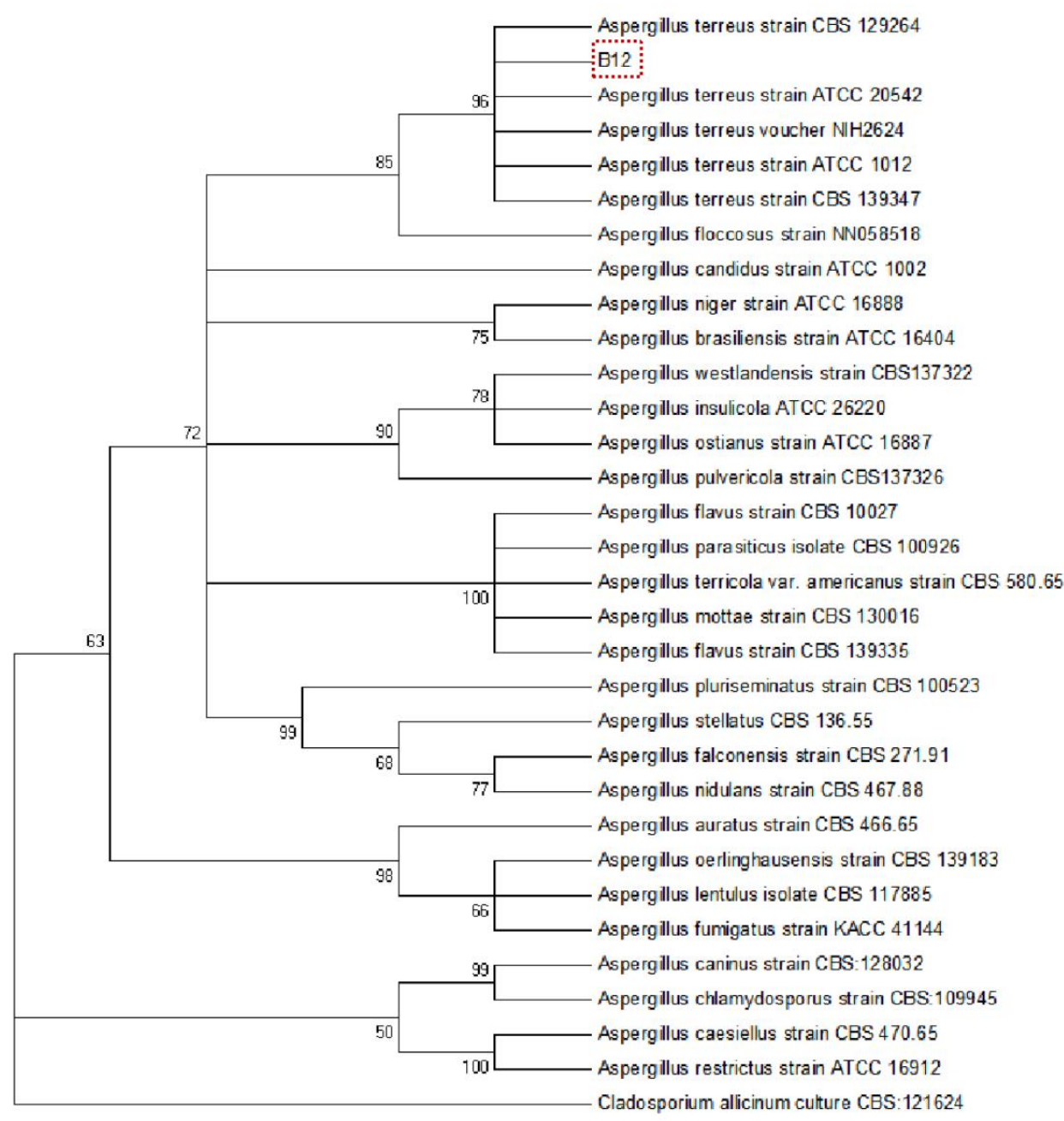

Figure 2. Identification of B12. A. Morphology of characterized strains, Colony 
and microscopic morphology after 5 days of incubation. From left to right: obverse colonies on MEA, reverse on MEA, conidiophores and conidiogenous at 40× magnification (scale bar $10 \mu \mathrm{m}$ ). $\quad$ B. Maximum likelihood tree of B12, Multiple sequence alignment was conducted using Clustal W (default settings), and phylogenetic relationships were based on ML analysis with 1000 bootstrap replications in MEGA7.

\section{Genome information of B12}

As genome-wide analysis provides first insights into the nature of microbial functioning [19], the genome of B12 was sequenced with a coverage of $173.87 \times$. The draft genome was assembled into a total size of $29.51 \mathrm{Mb}$, with a $\mathrm{G}+\mathrm{C}$ content of $52.31 \%$, composing 80 scaffolds. The average length of consensus contigs was $368922.8 \mathrm{bp}$ with an N50 of $1603970 \mathrm{bp}$. The protein-coding regions were predicted via Augustus software, resulting in a total of 10148 protein-coding genes with an average length of $1548.28 \mathrm{bp}$. The general genomic characteristics of strains B12 is listed in Table 1.

Table 1. General features of the B12 genomes

\begin{tabular}{ll}
\hline Genome & Value \\
\hline Assembly size (Mp) & 29.51 \\
$\mathrm{G}+\mathrm{C}(\%)$ & 52.31 \\
Assembled scaffolds & 80 \\
N50 length ( bp ) & 1603970 \\
average length ( bp ) & 368922.8 \\
predicted protein-coding genes & 10148 \\
average length predicted protein-coding genes & 1548.28 \\
average depth of reads cover & 173.87 \\
sequencing method & Illumina HiSeq \\
\hline
\end{tabular}

\section{Functional annotation}

As far as the structural-functional correlation is concerned, the function of genes, and the presumptive encoding products, can be predicted by sequence similarity[20]. Practically, comparative genomics on B12 genome yielded similar functional annotations in CAZy, KEGG and COG database: a large number of genes involve in metabolic pathways, indicating a vigorous potency in biochemical metabolism. According to the KEGG analyses, 4204 coding genes were annotated, assigned to 332 pathways, which could be classified into 6 functional categories: cellular processes, Environmental Information Processing, Genetic Information Processing, Human Diseases, Metabolism, Organismal Systems(Fig.3). Among the metabolic processes, 
besides the primary metabolism (e.g., amino acid, carbohydrate and lipid metabolism), the subcategory of SMs biosynthesis was conspicuous for the significant abundance and diversity(11.94\%), which underlined the potential biosynthetic capabilities in the strain.

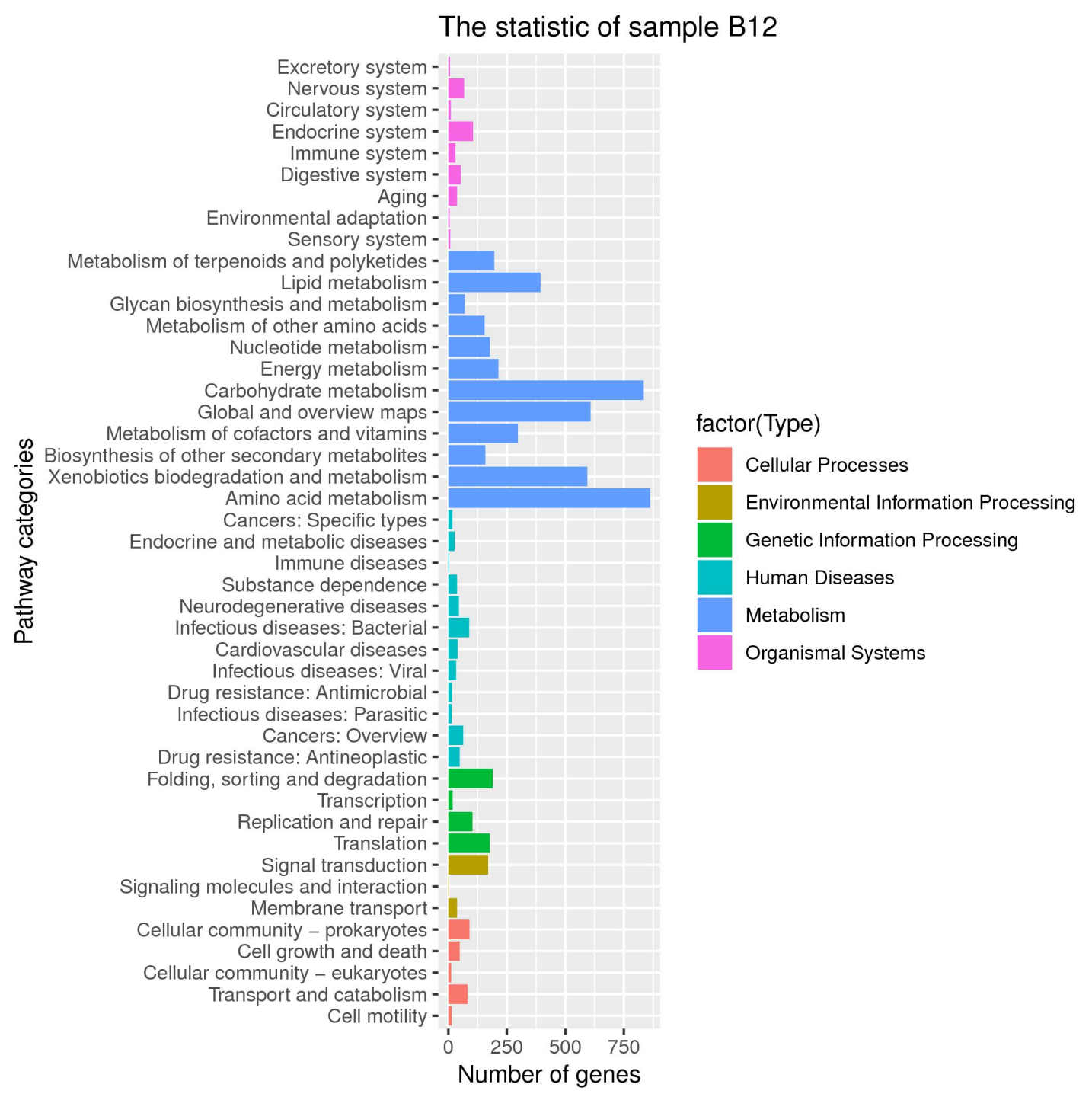

Figure 3. KEGG classifications of predicted coding genes in the genome of B12. Red: Cellular Processes; Brown: Environment Information Processing; Green: Genetic Information Processing; Cyan: Human Diseases; Blue: Metabolism; Purple: Organismal Systems.

Using CAZy functional classification, the global search assigned 1984 genes associated with carbohydrate active enzyme, accounting for $19.55 \%$ of the total protein-coding genes. The putative CAZymes comprised 6 groups: Carbohydrate-Binding Modules (CBMs), Auxiliary Activities (AAs), Carbohydrate Esterases (CEs), Polysaccharide Lyases (PLs), Glycosyl Transferases (GTs), Glycoside Hydrolases (GHs). Among those subfamilies, CBMs (27.92\%), GTs (26.66\%) and GHs (33.77\%) appeared as the majority contribution ( 88.36\%, Fig.4). 
Presumably, the multifarious CAZymes could be an accessible resource for nutrient acquisition and niche adaptation, in term of their potency and versatility in carbohydrates degradation and biotransformation[21].

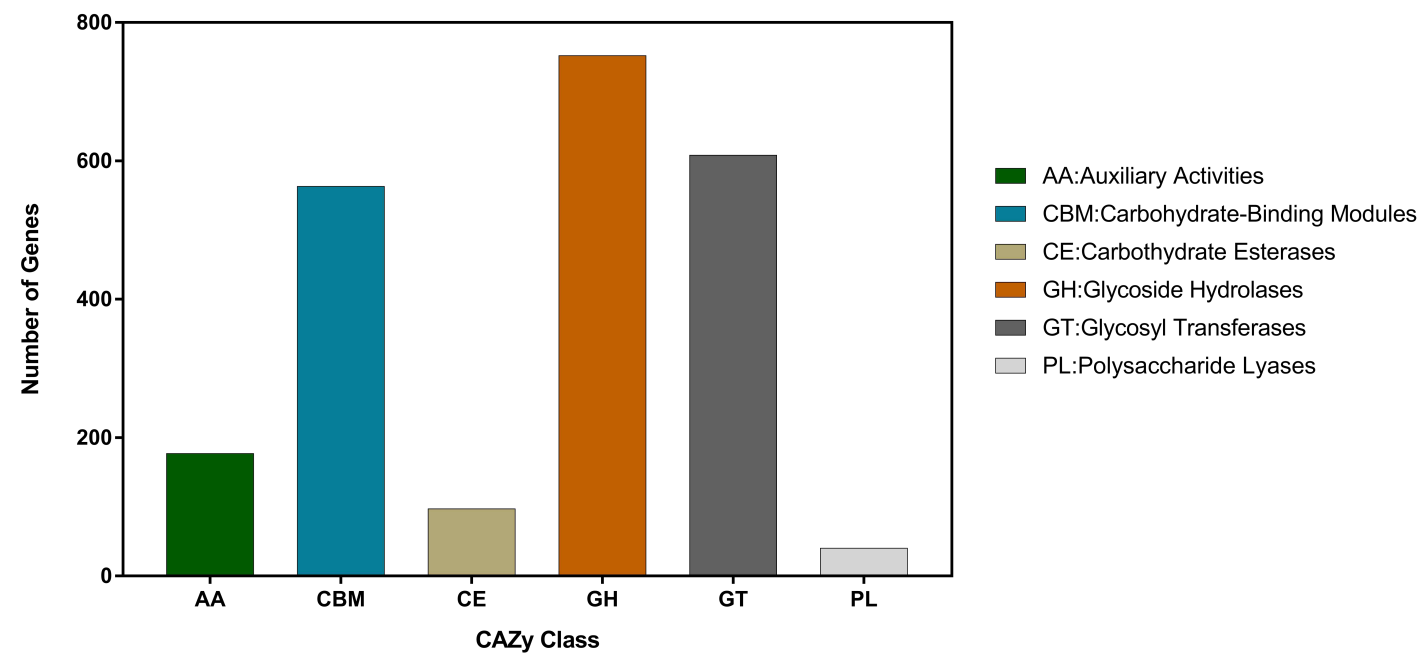

Figure 4. CAZy classifications of putative proteins in the genome of B12.

In COG database, the ortholog query assigned 6886 proteins, accounting for $67.86 \%$ of the total protein-coding genes. Concomitantly, the precedence of metabolism was verified by the considerable proportion (57.39\%) in functional categories (Fig.5), which was successively followed by poorly characterized function (26.20\%), information storage processing (15.93\%) and intracellular processes (13.64\%). Of note, in the metabolic patterns, the bioprocesses associated with SMs were evident, represented by the relative proportion of this category $(4.53 \%)$, which might confer a biochemical flexibility and adaptive superiority to the strain. 


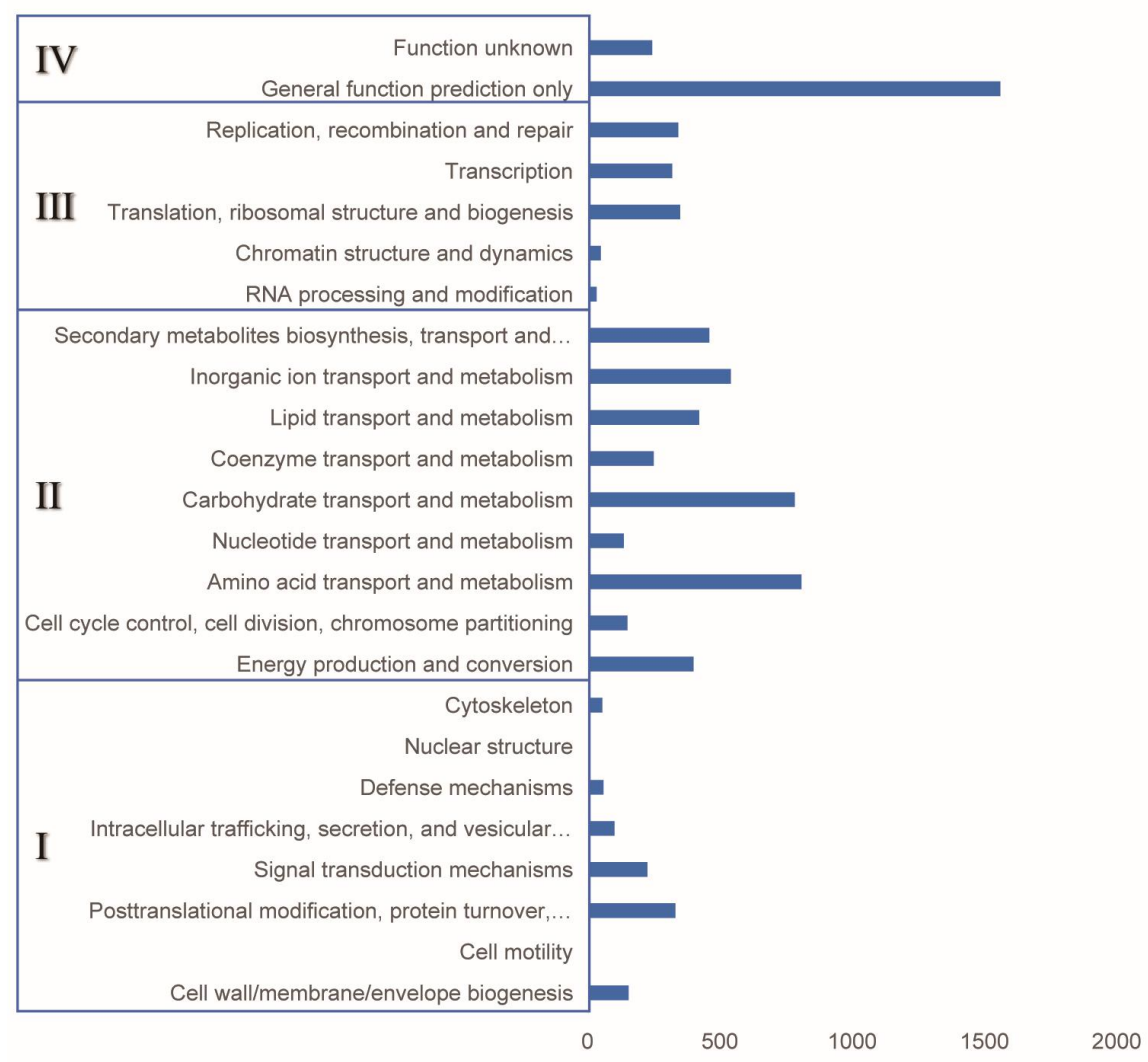

Figure 5. COG classifications of putative proteins in the genome of B12. I: intracellular processes; II: metabolism; III: information storage/processing; IV: poorly characterized function.

\section{Biosynthesis prediction of strain B12}

Different functional annotation systems integrated to highlight the biosynthetic potential in strain B12, possibly contributing to its antimicrobial activities. In fungal genomes, the genes that associated with SMs were often clustered, referred to as BGCs [22].

The speculative BGCs in B12 were predicted by antiSMASH database, forwarding 67 BGCs (Fig.6). In terms of biosynthetic pattern, the BGCs were classified into 10 types: NRPS (27), PKS (17), NRPS-indole hybrid (5), NRPS-T1PKS hybrid (5), NRPS-betalactone (1), NRPS-terpene hybrid (1), terpene (5), indole (4), betalactone (1), siderophore (1). 


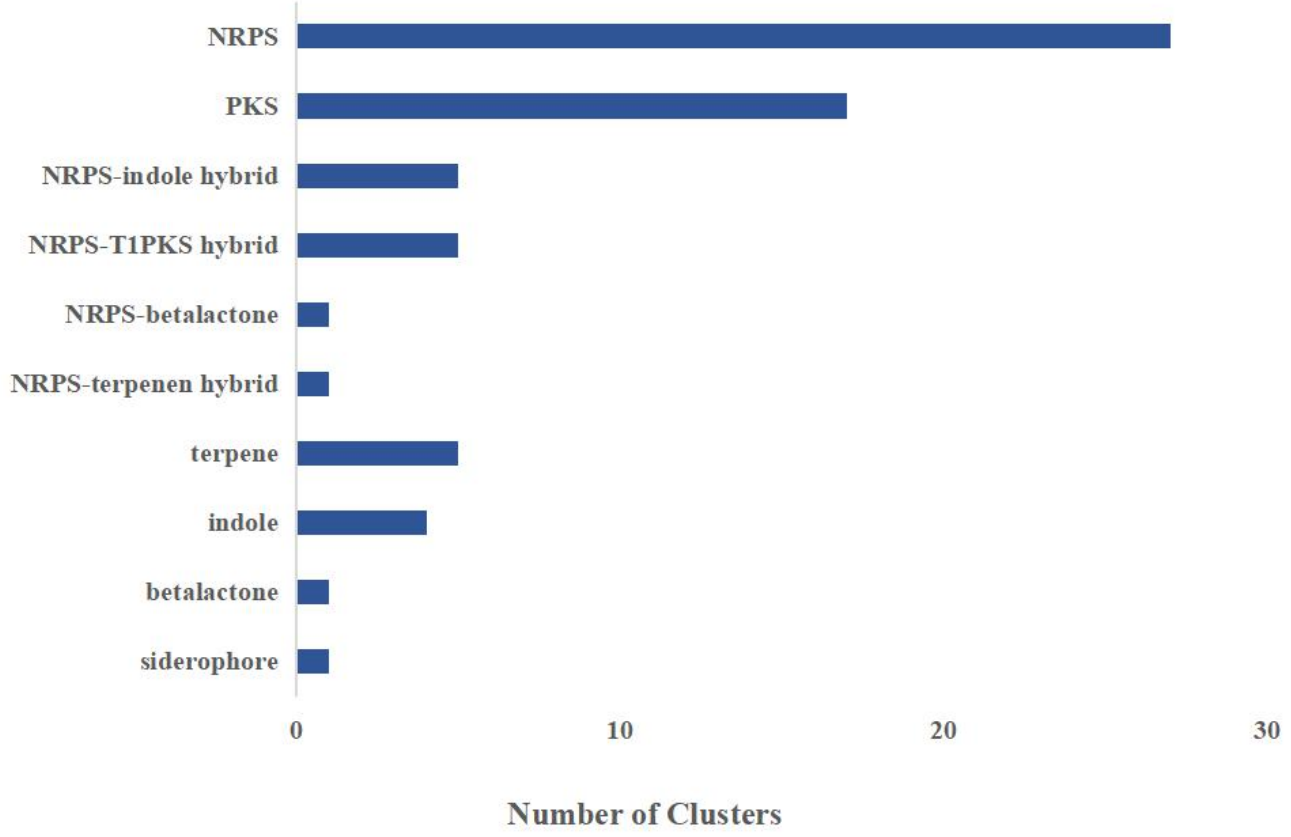

Figure 6. BGCs predicted in the B12 genomes based on antiSMASH

The fertile and multifarious BGCs, supposedly endorse the biosynthetic dexterity to produce novel chemical backbones or natural products, which certainly merits more exploration. Sequence alignment underscored 8 BGCs with $100 \%$ similarity to known BGCs, corresponding to various natural products, clavaric acid, dihydroisoflavipucine/ isoflavipucine, dimethylcoprogen, alternariol, aspterric acid and pyranonigrin E (Fig.7). In term of the structural-functional correlations, the chemical diversity of inferred SMs supposed to entail various roles, which have been demonstrated by cumulative reports, comprising antitumor (clavaric acid[23]) and antioxidative (pyranonigrin E[24]) agents, siderophores (dimethylcoprogen[25]), plant growth regulator (aspterric acid[26]) and phytotoxins (alternariol[27], dihydroisoflavipucine/ isoflavipucine[28]). Besides variance in the proposed products, the diversification of biosynthetic patterns was equally inspirational, involving T1PKS (Fig. 7 A-C), NRPS-PKS hybrid (Fig.7 D-E), NRPS (Fig.7 F), terpene (Fig.7 $\mathrm{G}-\mathrm{H}$ ), which might imply a pluripotent biochemical toolkit in this strain, as determined from the current limited genome-mining scope. 


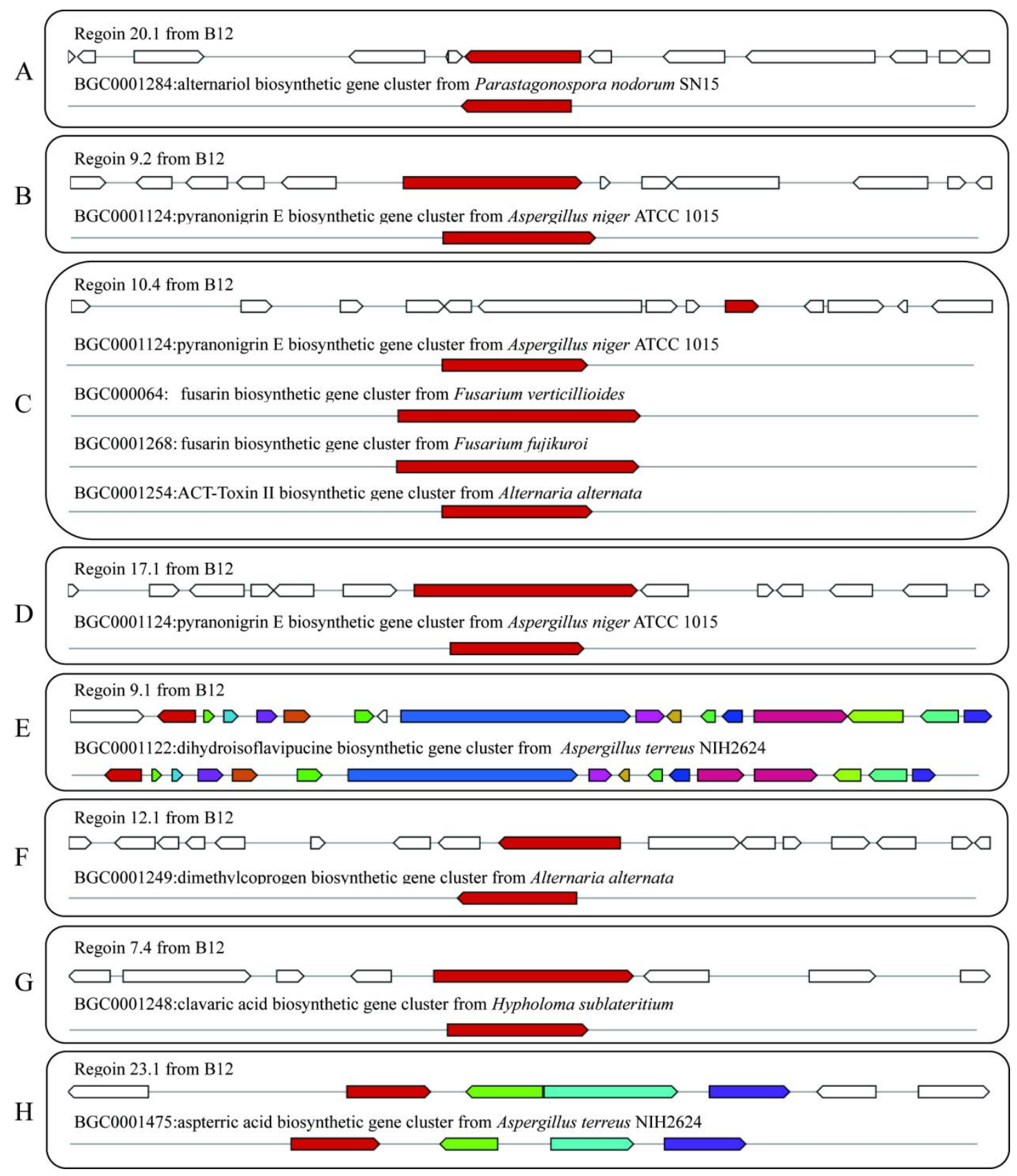

Figure 7. Schematic representation of B12 putative BGCs showing high similarity with genes from characterized BGCs. A-H. The upper part represents the BGC in B12, followed by the known BGCs in the MIBiG database.

In addition, a gene cluster homologous ( $44 \%)$ to the $\mathrm{BGC}$ of monacolin $\mathrm{K}$ (MK/lovastatin) was found in B12 genome (Fig.8 A). As the inhibitor of HMG CoA reductase[29], Lovastatin was originally obtained from a soil-derived Aspergillus terreus strain[30], and also produced by other fungal genera like Penicillium, Paecilomyces, Trichoderma, and Pleurotus ostreatus[31]. Despite the considerable similarity of genes organization and sequence identity $(>70 \%)$, the region in $\mathrm{B} 12$ exhibited an obvious different pattern from MK/lovastatin biosynthetic pathway, 
reflected by the absence of LovE and distinct truncation of LovF. Insofar as is known, LovE gene encodes a transcription factor that regulates $\mathrm{MK} /$ lovastatin gene cluster, whereas lovF encodes a diketide synthase (DKS), one of the two polyketide synthases involved in $\mathrm{MK} /$ lovastatin biosynthesis[32], underlying the indispensability of either part. Actually, we failed to obtain lovastatin from the fermented products of B12, appearing plausible from the perspective of biosynthetic mechanism.

$\mathbf{A}$

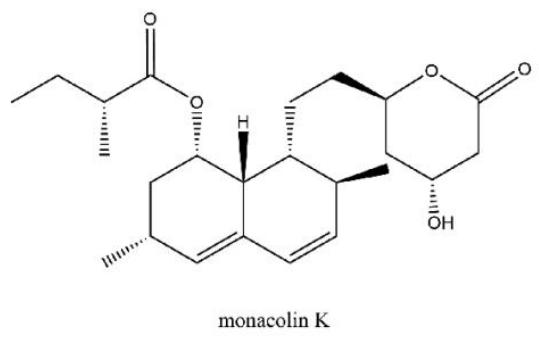

B Regoin 25.1 from B12

\begin{tabular}{lllll} 
& & \\
\hline Bdentifiers in Aspergillus terreus & Gene code & Product & Query cover & Identity \\
\hline AAD34550.1 & --- & esterase & 95 & 90 \\
AAD34551.1 & -- & unknown & -- & -- \\
AAD34552.1 & lovA & cytochrome P450 monooxygenase & 104 & 96 \\
AAD34553.1 & -- & unknown & 99 & 94 \\
AAD34554.1 & lovC & enoyl reductase & 114 & 86 \\
AAD34555.1 & lovD & transesterase & 100 & 99 \\
AAD34556.1 & -- & hydroxymethylglutaryl-coenzyme A reductase & 81 & 96 \\
AAD34557.1 & lovE & regulatory protein & -- & --- \\
AAD34558.1 & -- & unknown & -- & --- \\
AAD34559.1 & lovF & polyketide synthase & 100 & 96 \\
\hline
\end{tabular}

Figure 8. Putative monacolin K BGC in B12. A.The structure of monacolin K. B.The homologous genes of region 25.1 in B12 and terrein BGC known in Aspergillus terreus have been marked with the same color, and the missing genes are marked with red dotted boxes. Known BGCs description and amino acid homology (query cover and identity) were listed in the table.

\section{Chemical Isolation and Characterization of Metabolites}

To interpret the biosynthetic capacities of B12, ethanol extraction of the fermentated product was prepared for SMs isolation. Chemical separation and characterization have identified a range of metabolites differed in panel structure and relative abundance, including Terrein (1), Butyrolactone I(2), Terretonin E(3), Terretonin A(4), Acoapetaline B(5), epi-Aszonalenins A(6) (Fig 9). 

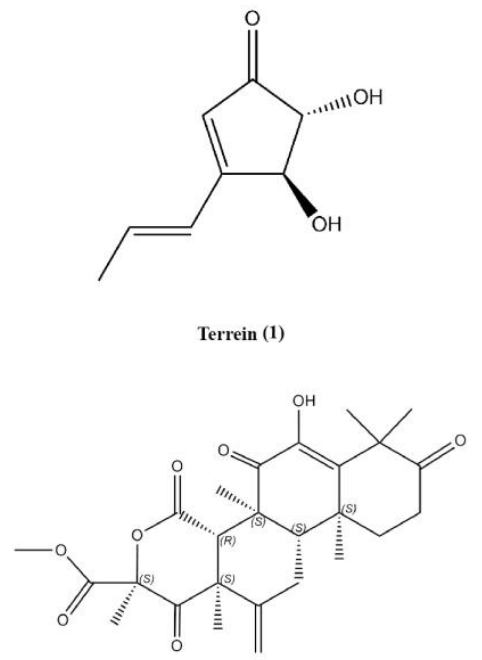

Terretonin A (4)

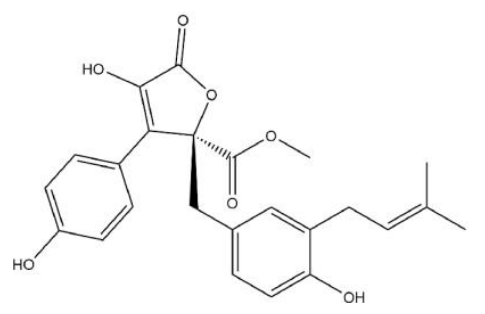

Butyrolactone I (2)

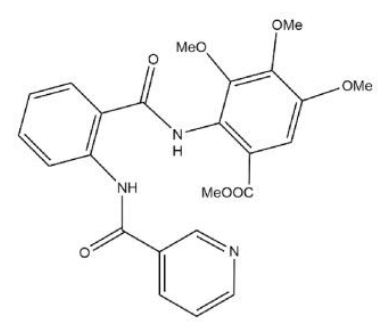

Acoapetaline B (5)

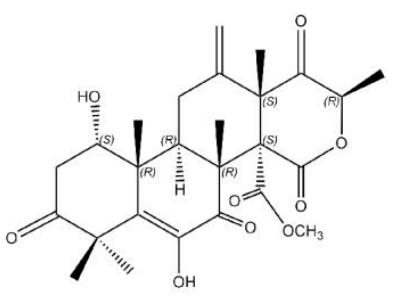

Terretonin E (3)

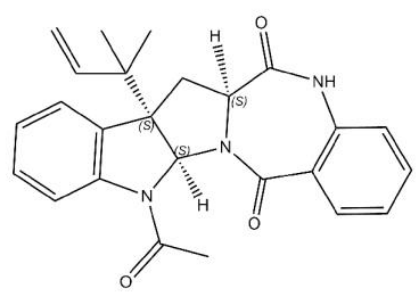

epi-Aszonalenin A (6)

Figure 9 The compounds was isolated from B12

Via chromatographic separation and HNMR determination, the compound terrein (1) was purified as yellow crystal needles, which is addressed as a A. terreus metabolite with ecological, antimicrobial, antiproliferative, and antioxidative activities[26] . Based on the chemical clue, a BGC presumably for terrein synthesis was mined in region 9.4 (Fig.10 B), demonstrating a high degree of amino acid sequence homology ( $>72 \%$ ) with the counterpart of $A$. terreus NIH2624[33]. However, despite the approximate gene composition and sequence identity, the assumed BGC was deprived of ter $\mathrm{H}-\mathrm{J}$ genes. In the case of terrein production, a gene locus comprised by terA-J and transcriptional regulator ter $\mathrm{R}$, was characterized to encode the biosynthetic process[26]. As ter $\mathrm{G}$ and ter $\mathrm{J}$ were depicted as MFS transporters, while function of terH-I was ambiguous, deletion of them putatively would lead to a diminution instead of abolition of terrein generation[33], which seemed to be substantiated in our study. Besides terrein, butyrolactones and terretonins are also frequently reported as typical SMs produced by A. terreus[34]. Hence, from the point of SMs production, the presence of those metabolites apparently complied with the species-specific descriptions on biosynthetic profiling. In contrast, as to the latter two, acoapetaline B (5) and epi-aszonalenins A (6), there was little information available, with limited clues in the original organisms and structural characterizations. It was reported that acoapetalines were isolated from plants $[35,36]$, while epi-aszonalenins derived from A. novofumigatus[37], ostensibly divergent from $A$. terreus. The identification of those compounds in strain B12 might be ascribed to the plentiful (33.85\%) uncharacterized BGCs to some extent, in term of the pendent and elusive insight of the biosynthetic processes and corresponding products in A. terreus [34], which awaits more advance of genome mining and genetic approaches. 
$\mathbf{A}$

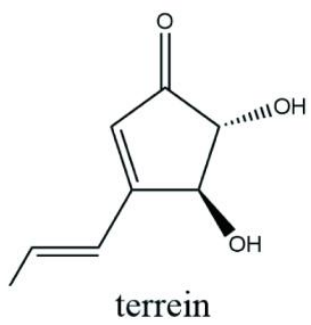

B

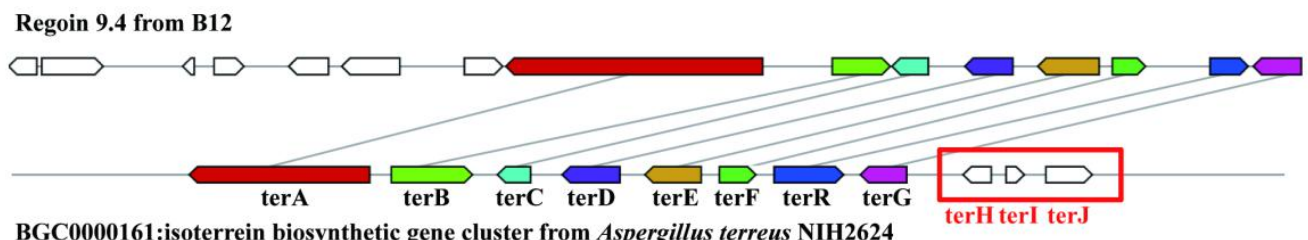

BGC0000161:isoterrein biosynthetic gene cluster from Aspergillus terreus NIH2624

\begin{tabular}{|c|c|c|c|c|}
\hline Identifiers in Aspergillus terreus $\mathrm{NIH} 2624$ & Gene code & Description & Query cover & Identity \\
\hline ATEG_00135 & terJ & major facility transporter (MFS) & --- & --- \\
\hline ATEG_00136 & terI & $\begin{array}{l}\text { protein of the superfamily of bleomycin resisitance } \\
\text { protein, glyoxalase I, and type I ring-cleaving } \\
\text { dioxygenanses }\end{array}$ & --- & --- \\
\hline ATEG_00137 & terH & NAD dependent epimerase/dehydratase & --- & -- \\
\hline ATEG_00138 & terG & major facility transporter (MFS) & $100 \%$ & $100 \%$ \\
\hline ATEG_00139 & terR & $\mathrm{Zn}_{2} \mathrm{Cys}_{6}$ transcriptional regulator & $99 \%$ & $72 \%$ \\
\hline ATEG_00140 & terF & protein with kelch motif & $123 \%$ & $80 \%$ \\
\hline ATEG_00141 & terE & multicopper oxidase & $94 \%$ & $91 \%$ \\
\hline ATEG_00142 & terD & FAD-dependent monooxygenase & $99 \%$ & $99 \%$ \\
\hline ATEG_00143 & terC & FAD-dependent monooxygenase & $144 \%$ & $68 \%$ \\
\hline ATEG_00144 & terB & $\begin{array}{l}\text { DH-KR, multidomain protein with dehydratase and } \\
\text { ketoreductase function }\end{array}$ & $74 \%$ & $73 \%$ \\
\hline ATEG_00145 & terA & non-reducing polyketide synthase (NR-PKS) & $84 \%$ & $97 \%$ \\
\hline
\end{tabular}

Figure 8. Putative Terrein BGC in B12. A.The structure of Terrein. B.The homologous genes of region 25.1 in B12 and terrein BGC known in Aspergillus terreus have been marked with the same color, and the missing genes are marked with red dotted boxes. B.Known BGCs description and amino acid homology (query cover and identity) were listed in the table.

\section{Discussion}

Aspergillus terreus, widely distributed in terrene and marine environment, has become a prolific producer of numerous bioactive agents, such as lovastatin, sulochrin, terrein, itaconic acid, etc[2]. In recent years, this fungus derived from ocean became more attractive, as concerning the structurally unique or biologically active metabolites[38]. In this study, an A. terreus strain B12 was isolated from sponges in the South China, which promisingly demonstrated broad bacteriostatic effects against a range of pathogenic bacteria. The whole genome was sequenced followed by comparative genomics analysis and functional annotations, leading to a total of 67 putative BGCs. 
However, only a fraction of putative BGCs (18) possessed a high sequence similarity $(\geqslant 50 \%)$ with annotated BGCs in the MIBiG database, in contrast with a variety of uncharacterized gene clusters, conjecturally which might provide a biosynthetic blueprint for the products diversification in the strain. The speculation was partially confirmed by chemical separation, which identified several compounds, consisting of SMs commonly in A. terreus (terrein, butyrolactone I and terretonins) or not (acoapetaline and epi-aszonalenins).

Besides the excellent ecological adaptability, A. terreus is an illustrious strain in the industrial production of lovastatin, a valuable cholesterol-lowering agent for hyperlipidemia treatment[39]. Lovastatin inhibits hydroxyl methyl glutaryl coenzyme A reductase (HMG CoA), the key enzyme of cholesterol biosynthesis, which is supposed as an adaptive strategy to halter fungal ergosterol generation, required for the maintenance of cytoplasmic membrane integrity[40, 41]. The lovastatin biosynthesis is encoded by a BGC (lov) containing 18 genes, among which, five genes $(\operatorname{lov} \mathrm{A}, \mathrm{B}, \mathrm{C}, \mathrm{D}$, and $\mathrm{F})$ have been identified to encode essential enzymes, while lovE acts as transcription factor, positively controlling the biogenic route[42, 43]. However, as for the homogenous $\mathrm{BGC}$ within $\mathrm{B} 12$ genome, the deletion of $\operatorname{lov} \mathrm{E}$ and a significant truncation of $l o v \mathrm{~F}$ were detected, which assumedly would abolish lovastatin production. In terms of the synthetic logic of lovastatin, which actually provides a biochemical aid for fungal self-protection[44], the acquisition of lov BGC by horizontal gene transfer might actually depend on the niche competitive status. The biosynthetic impuissance due to gene deficits had been reported in an endophytic $A$. terreus strain[45], which possibly should ascribe to the primitive symbiotic environments of the strain above-mentioned or B12, for the comparable but diverse philosophy.

The substantial biosynthetic potential was highlighted by genome mining within B12, depicted by various uncharacterized BGCs, which requested the corresponding linkage with the product. However, compared with lovastatin, many other synthetic processes still remains elusive, even within the best understood genome of $A$. terreus (strain NIH 2624)[34]. For instance, from fermented extract of B12, terretonins and butyrolactone I had been isolated, which were adopted as major metabolites of $A$. terreus and physiological regulators in response to ecological competition [46, 47]. It had been proposed that a compact cluster (trt) was responsible for terretonin biosynthesis, although conversion process to the end product (terretonin) remained ambiguous, which might involve other genes in different loci[48]; While a NRPS-like gene btyA was presumed to encode the core enzyme for the backbone of 
$\gamma$-butyrolactone in A. terreus[49]. Whereas, according to the sequence homology, the attempt to assign those products to possible BGCs within the B12 genome led to failure, probably due to currently obscure understanding on secondary metabolic pathways, especially given the possibility of isozymes or orthologs presented in the unknown BGCs, which could lead to partly overlapping biosynthetic routes[50, 51].

\section{Conclusion}

In our study, the combination of genome mining and compound separation, although far from comprehensive, have underscored the metabolic potencies of the A. terreus strain B12, which might ascribe to the exclusive biological plasticity for niche acclimatization. The peculiar metabolites and BGCs, either characterized or not, presumptively contribute to the defensive mechanisms and survival strategies for the strain, in light of the harshness and competitiveness of marine habitats. Our work shed more light on the genetic and biological profiling of marine-derived A. terreus, which might facilitate further investigation and development of the valuable microorganism.

\section{Acknowledgements}

This work was supported by funds from the National Key R\&D Program of China (2018YFC0311000), the Priority Academic Program Development of the Jiangsu Higher Education Institutions (PAPD).

\section{Reference}

1. Shin, H.J.M.d., Natural Products from Marine Fungi. 2020. 18(5).

2. Rateb, M.E. and R.J.N.P.R. Ebel, Secondary metabolites of fungi from marine habitats. 2011. 28(2): p. 290-344.

3. Johannes, I.J.M.D., Natural Products from Marine Fungi-Still an Underrepresented Resource. 2016. 14(1).

4. Taylor, M., et al., Sponge-associated microorganisms: evolution, ecology, and biotechnological potential. 2007. 71(2): p. 295-347.

5. H?Ller, U., et al., Fungi from marine sponges: diversity, biological activity and secondary metabolites. 2000. 104(11): p. 1354-1365.

6. Gao, Y., et al., Genome sequence of Aspergillus flavus A7, a marine-derived fungus with antibacterial activity. 2020.

7. Leck, A.J.C.E.H., Preparation of Lactophenol Cotton Blue Slide Mounts. 1999. 12(30): p. 24.

8. Lee, S., J.J.M.b. Taylor, and evolution, Phylogeny of five fungus-like protoctistan Phytophthora species, inferred from the internal transcribed spacers of ribosomal DNA. 1992. 9(4): p. 636-53.

9. Hinrikson, H., et al., Assessment of ribosomal large-subunit D1-D2, internal transcribed spacer 1 , and internal transcribed spacer 2 regions as targets for molecular identification of medically important Aspergillus species. 2005. 43(5): p. 2092-103. 
10. Zerbino, D. and E.J.G.r. Birney, Velvet: algorithms for de novo short read assembly using de Bruijn graphs. 2008. 18(5): p. 821-9.

11. Boetzer, M., et al., Scaffolding pre-assembled contigs using SSPACE. 2011. 27(4): p. 578-9.

12. Boetzer, M. and W.J.G.b. Pirovano, Toward almost closed genomes with GapFiller. 2012. 13(6): p. R56.

13. Blin, K., et al., antiSMASH 5.0: updates to the secondary metabolite genome mining pipeline. 2019. 47: p. W81-W87.

14. Stanke, M., et al., AUGUSTUS: ab initio prediction of alternative transcripts. 2006. 34: $\mathrm{p}$. W435-9.

15. Consortium, G.O.J.N.A.R., The Gene Ontology (GO) database and informatics resource. 2004(suppl_1):p.suppl_1.

16. Kanehisa, M., et al., New approach for understanding genome variations in KEGG. 2019. 47: p. D590-D595.

17. Tatusov, R., et al., The COG database: an updated version includes eukaryotes. 2003. 4: p. 41.

18. Schoch, C., et al., Nuclear ribosomal internal transcribed spacer (ITS) region as a universal DNA barcode marker for Fungi. 2012. 109(16): p. 6241-6.

19. Zhu, B., et al., Multi-omics analysis of niche specificity provides new insights into ecological adaptation in bacteria. 2016.

20. Alberts, B., et al., Integrins - Molecular Biology of the Cell - NCBI Bookshelf. 2002.

21. Chettri, D., A.K. Verma, and A.K.J.B.R. Verma, Innovations in CAZyme gene diversity and its modification for Biorefinery applications. 2020: p. e00525.

22. Bok, J., et al., Genomic mining for Aspergillus natural products. 2006. 13(1): p. 31-7.

23. Godio, et al., A Squalene Epoxidase Is Involved in Biosynthesis of Both the Antitumor Compound Clavaric Acid and Sterols in the Basidiomycete H. sublateritium. 2007.

24. Shindo, Riko, and N.J.T.J.o.A.A.I. Journal, Studies on pyranonigrins-isolation of pyranonigrin $E$ and biosynthetic studies on pyranonigrin A. 2014. 67(2): p. 179-181.

25. Jalal, M., S.K. Love, and D.J.B.o.M. Helm, $N \alpha$-Dimethylcoprogens Three novel trihydroxamate siderophores from pathogenic fungi. 1988. 1(1): p. 4-8.

26. Zaehle, C., et al., Terrein biosynthesis in Aspergillus terreus and its impact on phytotoxicity. 2014. 21(6): p. 719-31.

27. Solhaug, A., et al., Mechanisms of Action and Toxicity of the Mycotoxin Alternariol: A Review. 2016.

28. Brock, H.M.J.C. and Biology, Multifactorial Induction of an Orphan PKS-NRPS Gene Cluster in Aspergillus terreus. 2011.

29. Alberts, et al., Mevinolin: a highly potent competitive inhibitor of hydroxymethylglutaryl-coenzyme A reductase and a cholesterol-lowering agent. 1980. 77(7): p. 3957-3961.

30. Mulder, K., et al., Lovastatin production: From molecular basis to industrial process optimization. 2015. 33: p. 648-65.

31. Miranda, R.U., et al., Reactive oxygen species regulate lovastatin biosynthesis in Aspergillus terreus during submerged and solid-state fermentations. 2014. 118(12).

32. Barrios-González, J., et al., New knowledge about the biosynthesis of lovastatin and its production by fermentation of Aspergillus terreus. 2020. 104(21): p. 8979-8998. 
33. Yin, Y., et al., Polyketides in Aspergillus terreus: biosynthesis pathway discovery and application. 2016. 100(18): p. 7787-7798.

34. Guo, C. and C.J.F.i.m. Wang, Recent advances in genome mining of secondary metabolites in Aspergillus terreus. 2014. 5: p. 717.

35. Hu, Z.X., et al., Seven new anthranilamide derivatives from Aconitum apetalum. 2018.

36. Xie, X.L., et al., A new prenylated coumarin and a new anthranilamide derivative from Evodia lepta. 2019: p. 1-5.

37. Rank, C., et al., epi-Aszonalenins A, B, and C from Aspergillus novofumigatus. 2006. 47(34): $p$. 6099-6102.

38. Zhang, H., Z. Zhao, and H.J.M.d. Wang, Cytotoxic Natural Products from Marine Sponge-Derived Microorganisms. 2017. 15(3).

39. Tobert, J.J.N.r.D.d., Lovastatin and beyond: the history of the HMG-CoA reductase inhibitors. 2003. 2(7): p. 517-26.

40. Rodrigues, M.J.m., The Multifunctional Fungal Ergosterol. 2018. 9(5).

41. Keller, N.J.N.c.b., Translating biosynthetic gene clusters into fungal armor and weaponry. 2015. 11(9): p. 671-7.

42. Campbell, C. and J.J.B. Vederas, Biosynthesis of lovastatin and related metabolites formed by fungal iterative PKS enzymes. 2010. 93(9): p. 755-63.

43. Kennedy, J., et al., Modulation of polyketide synthase activity by accessory proteins during lovastatin biosynthesis. 1999. 284(5418): p. 1368-72.

44. Zhgun, A.A., et al., The Role of LaeA and LovE Regulators in Lovastatin Biosynthesis with Exogenous Polyamines in Aspergillus terreus. 2019. 55(6): p. 639-648.

45. Savitha, J., S.D. Bhargavi, and V.K. Praveen, Bioinformatic Comparative Analysis of Lovastatin Gene Cluster in Endophytic Fungi and a Soil Fungus, Aspergillus terreus. 2014.

46. Schimmel, T.G., et al., Effect of butyrolactone I on the producing fungus, Aspergillus terreus. 1998. 64(10): p. 3707-12.

47. Louis, B., et al., Invasive Aspergillus terreus morphological transitions and immunoadaptations mediating antifungal resistance. 2017. 10: p. 425-436.

48. Guo, C.J., et al., Molecular Genetic Characterization of a Cluster in A. terreus for Biosynthesis of the Meroterpenoid Terretonin. 2012. 14(22).

49. Guo, C.J., et al., Application of an efficient gene targeting system linking secondary metabolites to their biosynthetic genes in Aspergillus terreus. 2013. 15(14): p. 3562-3565.

50. Hossain, A.H., et al., Rewiring a secondary metabolite pathway towards itaconic acid production in Aspergillus niger. 2016. 15(1): p. 130.

51. Spatial regulation of a common precursor from two distinct genes generates metabolite diversity \%J Chemical Science. 2015. 6(10): p. 5913-5921. 


\section{Figures}

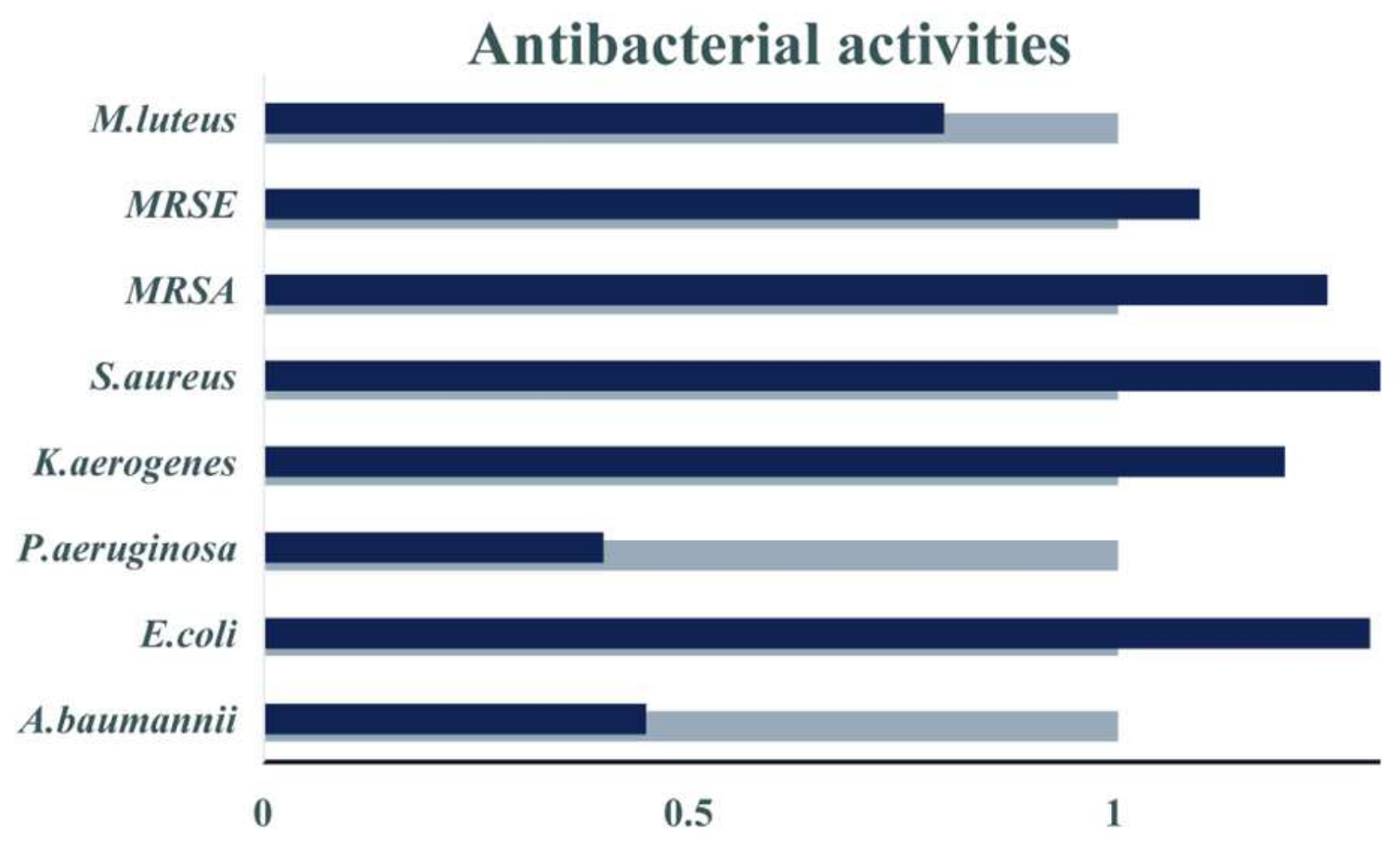

Figure 1

The antibacterial activities of the extractive of B12. The standard antibiotics ( $0.1 \mathrm{mM}$ chloramphenicol) were used as a positive control, $\mathrm{MeOH}$ was used as a vehicle. The diameters (in $\mathrm{mm}$ ) of the inhibition zone were recorded to estimate antimicrobial activities, which were expressed by the ratio of the inhibition

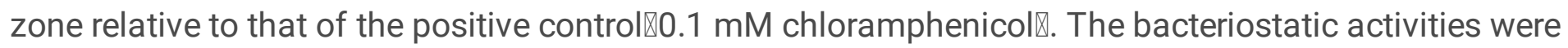
considered strong if the ratio was greater than 1.0, moderate when the scale was between 0.5 and 1 , and weak if it was less than 0.5 .the blue bar indicated as 1 , the black-blue indicated as the ratio of the inhibition zone relative to that of the positive control. E. coli: Escherichia coli; P. aeruginosa: Pseudomonas aeruginosa; K. aerogenes: Klebsiella aerogenes; MRSA: methicillin-resistant Staphylococcus aureus; MRSE: methicillin-resistant Staphylococcus epidermidis; M. luteus: Micrococcus luteus; A. baumannii: Acinetobacter baumannii. 
A
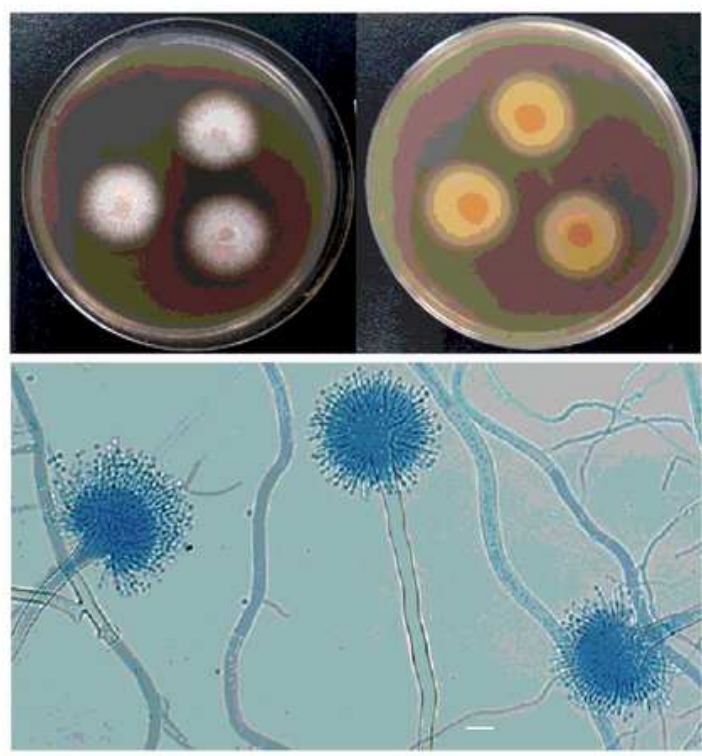

B

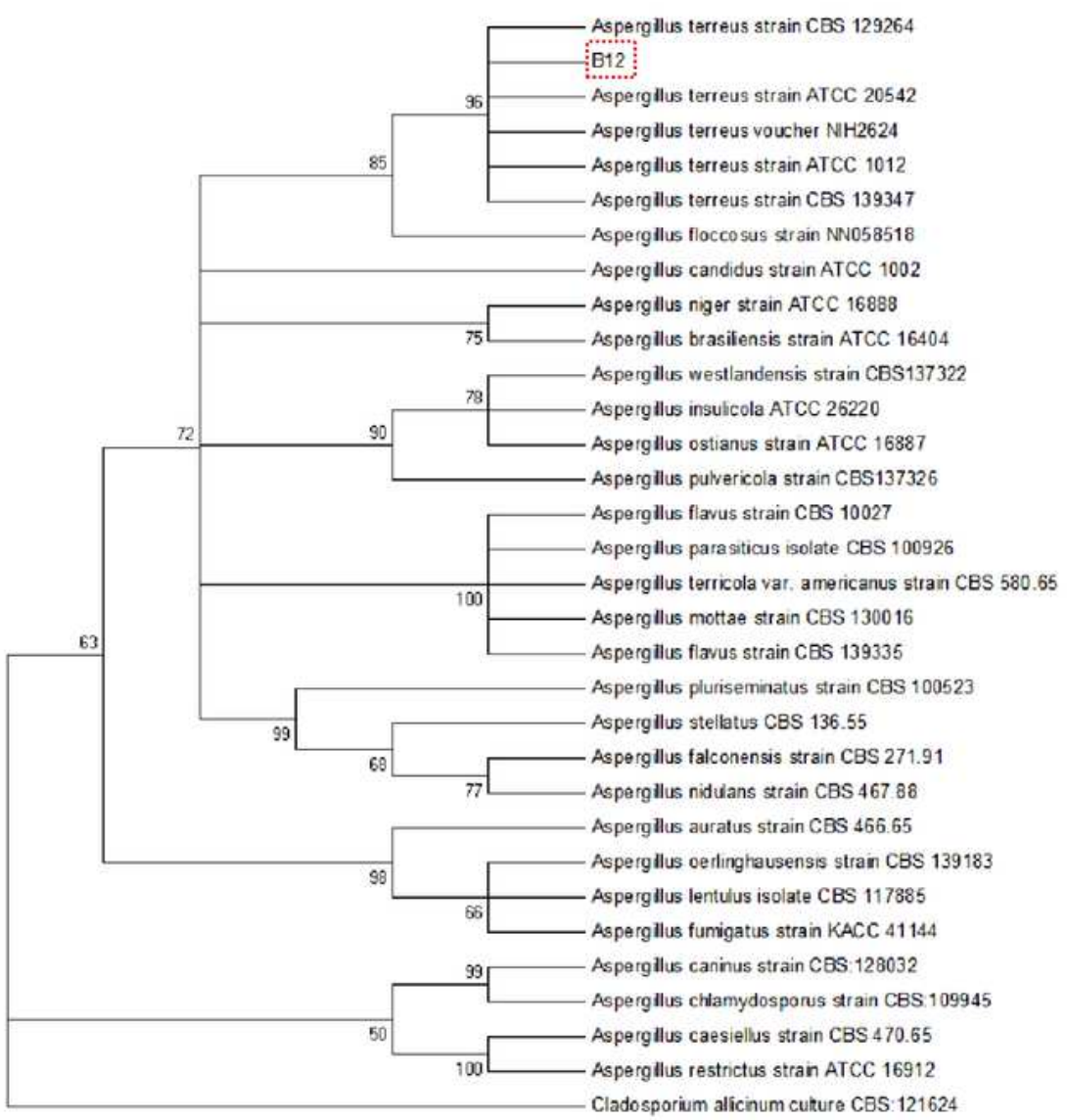

Figure 2

Identification of B12. A. Morphology of characterized strains, Colony and microscopic morphology after 5 days of incubation. From left to right: obverse colonies on MEA, reverse on MEA, conidiophores and conidiogenous at 40x magnification (scale bar $10 \mu \mathrm{m}$ ). B. Maximum likelihood tree of B12, Multiple sequence alignment was conducted using Clustal W (default settings), and phylogenetic relationships were based on ML analysis with 1000 bootstrap replications in MEGA7. 


\section{The statistic of sample B12}

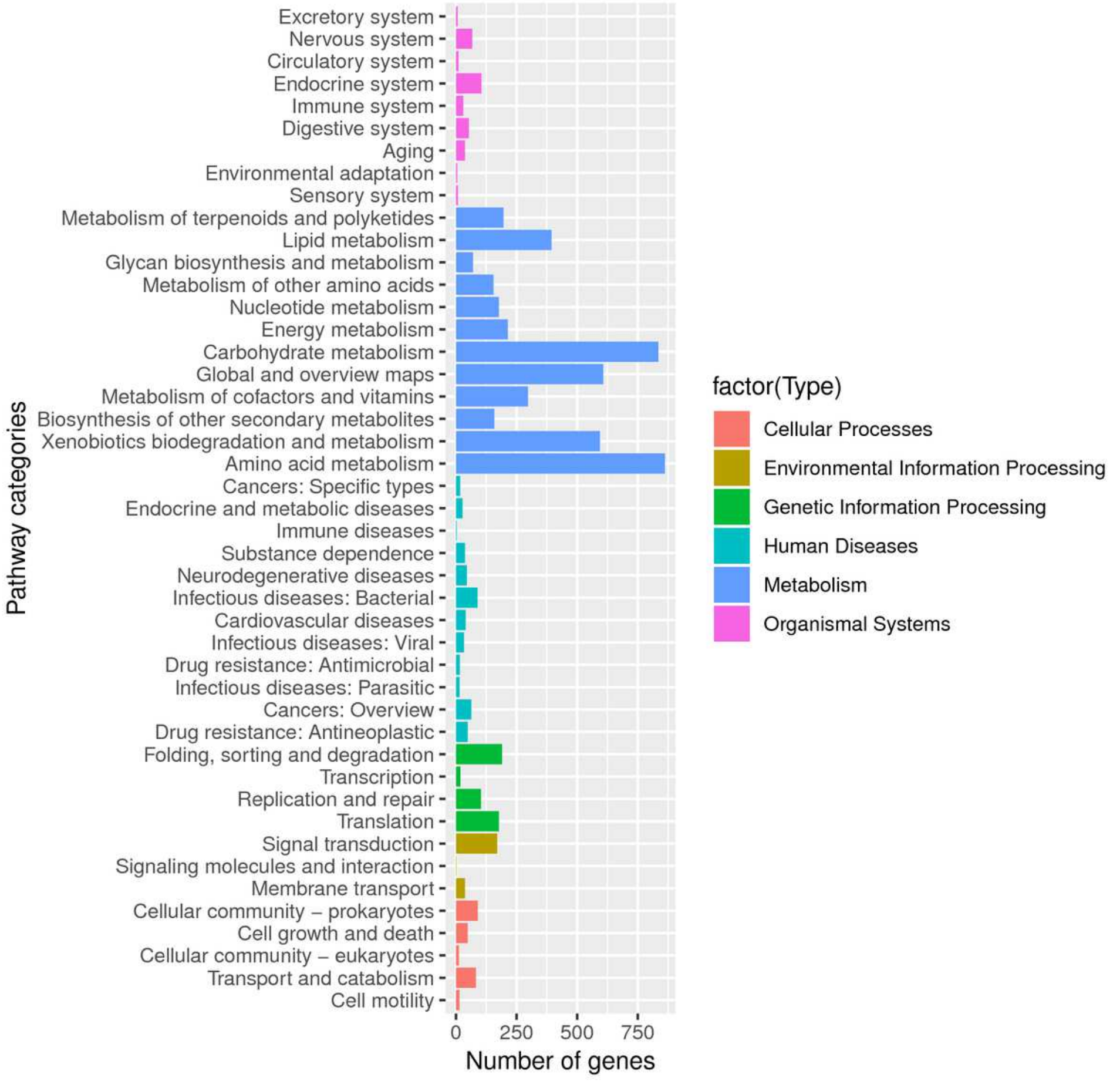

\section{Figure 3}

KEGG classifications of predicted coding genes in the genome of B12. Red: Cellular Processes; Brown: Environment Information Processing; Green: Genetic Information Processing; Cyan: Human Diseases; Blue: Metabolism; Purple: Organismal Systems. 


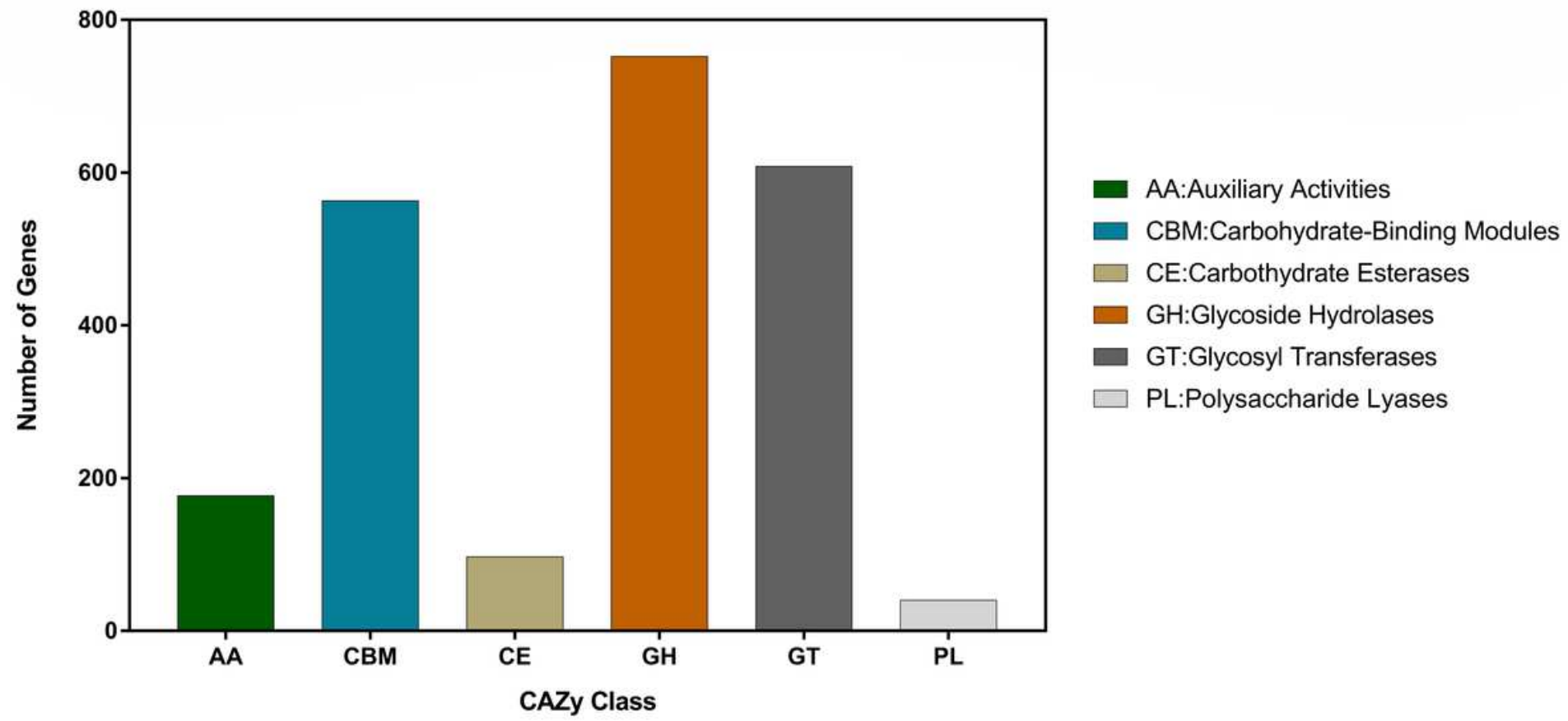

Figure 4

CAZy classifications of putative proteins in the genome of B12. 


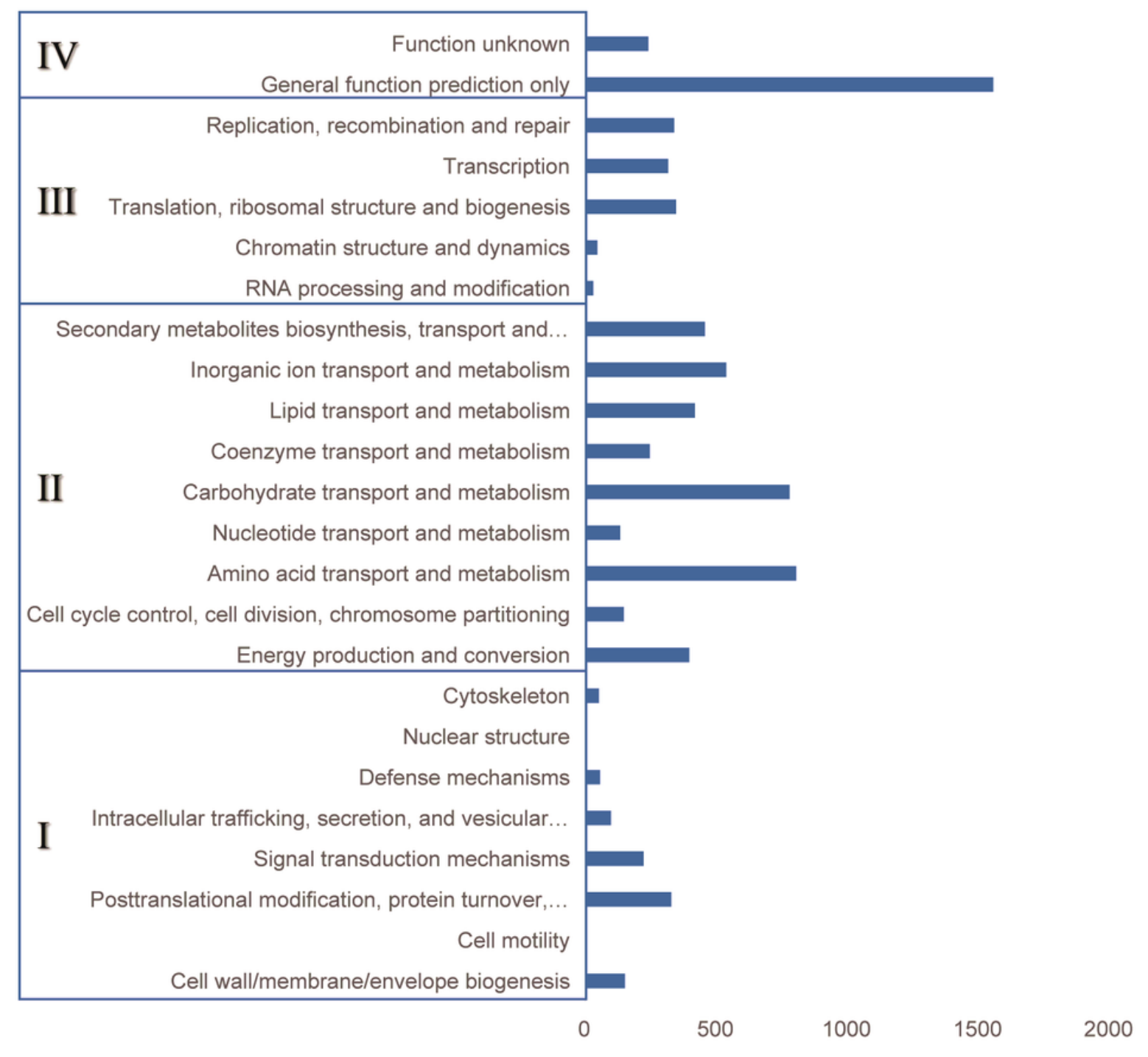

Figure 5

COG classifications of putative proteins in the genome of B12. I: intracellular processes; II: metabolism; III: information storage/processing; IV: poorly characterized function. 


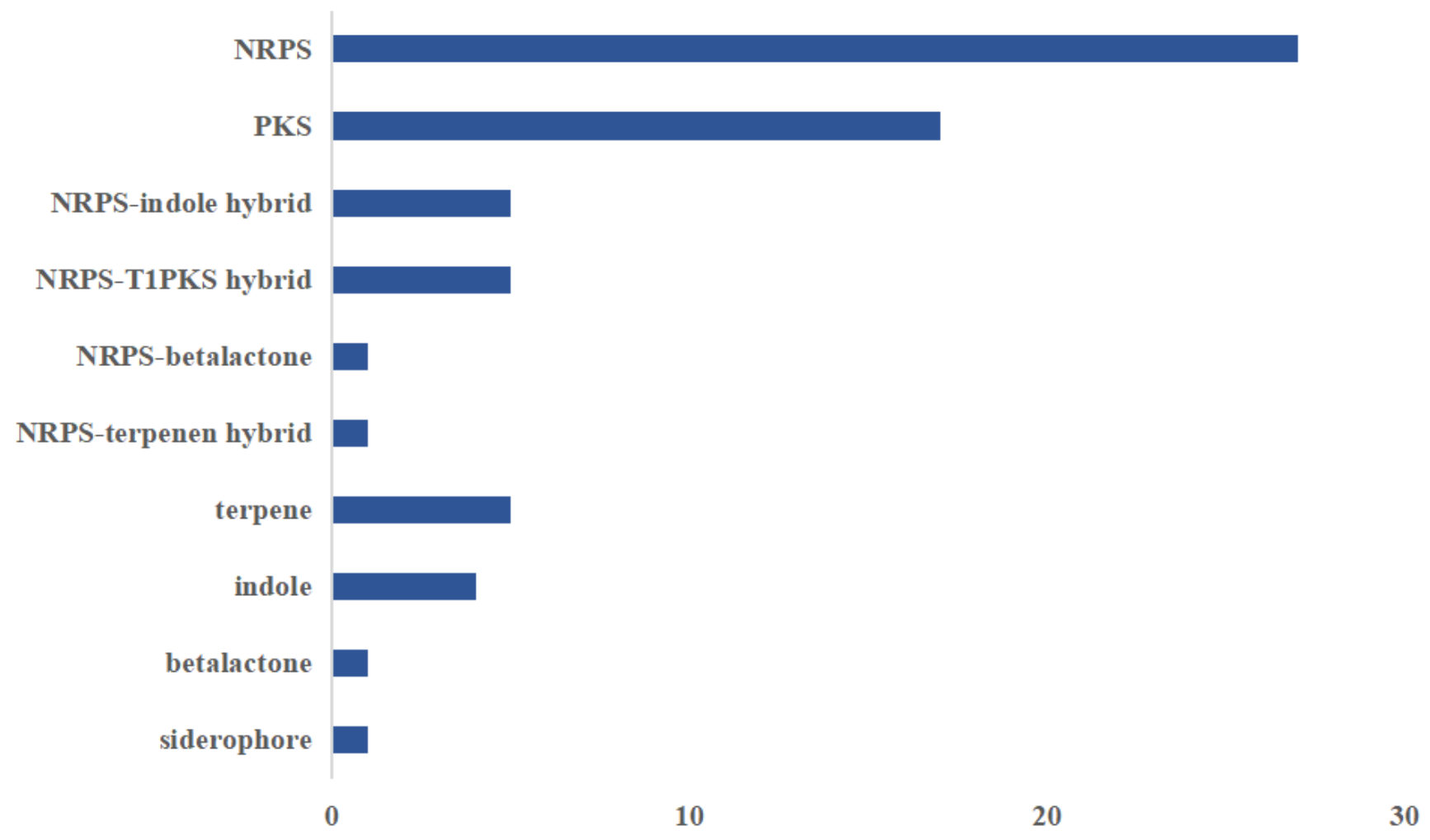

Number of Clusters

Figure 6

BGCs predicted in the B12 genomes based on antiSMASH 
Regoin 20.1 from B12

$\triangleright \square \square$

BGC0001284:alternariol biosynthetic gene cluster from Parastagonospora nodorum SN15

BGC0001284:alterariol biosynthetic gene cluser firom Parastagonospor

Regoin 9.2 from B12

B

BGC0001124:pyranonigrin E biosynthetic gene cluster from Aspergillus niger ATCC 1015

$\square$

Regoin 10.4 from B12

BGC0001124:pyranonigrin E biosynthetic gene cluster from Aspergillus niger ATCC 1015

C

BGC000064: fusarin biosynthetic gene cluster from Fusarium verticillioides

BGC0001268: fusarin biosynthetic gene cluster from Fusarium fuiikuroi

BGC0001254:ACT-Toxin II biosynthetic gene cluster from Alternaria alternata

$\longrightarrow$

Regoin 17.1 from B12

$D \square \square \square \square \square \square \square \square \square \square$

D BGC0001124:pyranonigrin E biosynthetic gene cluster from Aspergillus niger ATCC 1015

$\square$
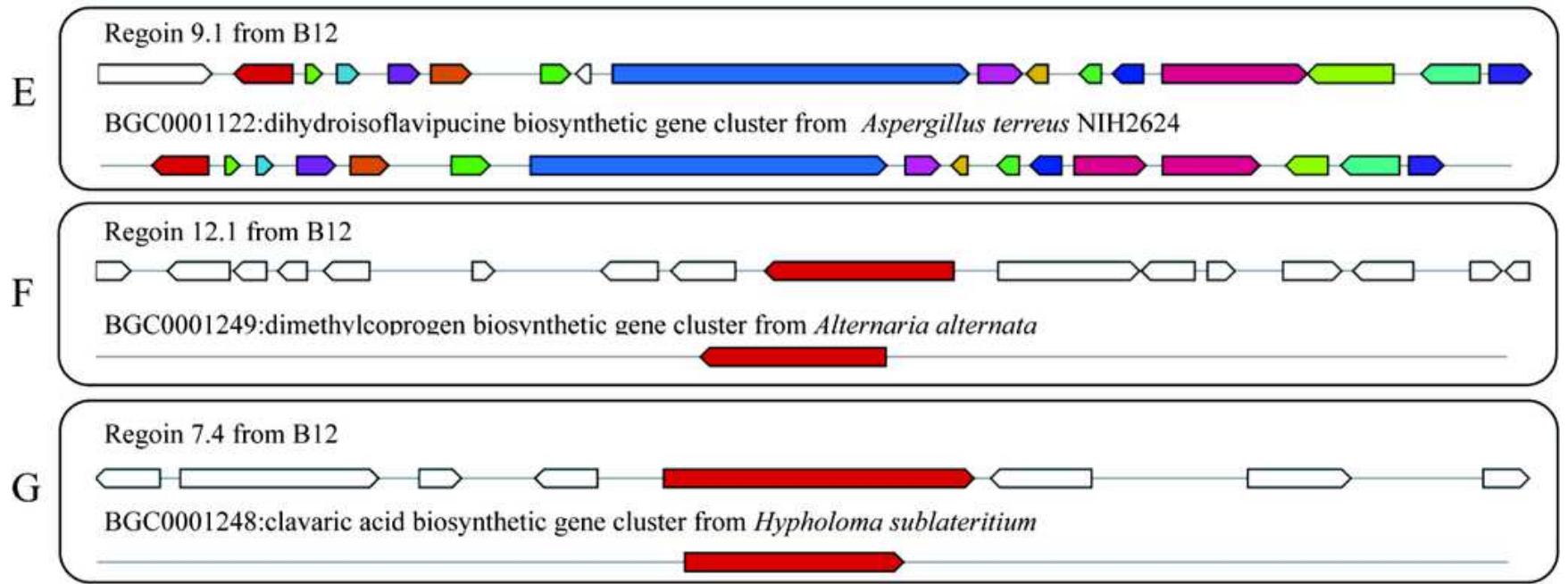

$\mathrm{H}$

Regoin 23.1 from B12

$\longrightarrow \square \longrightarrow$

BGC0001475:aspterric acid biosynthetic gene cluster from Aspergillus terreus NIH2624

$\square \square$

Figure 7

Schematic representation of B12 putative BGCs showing high similarity with genes from characterized BGCs. A-H. The upper part represents the BGC in B12, followed by the known BGCs in the MIBiG database. 
A

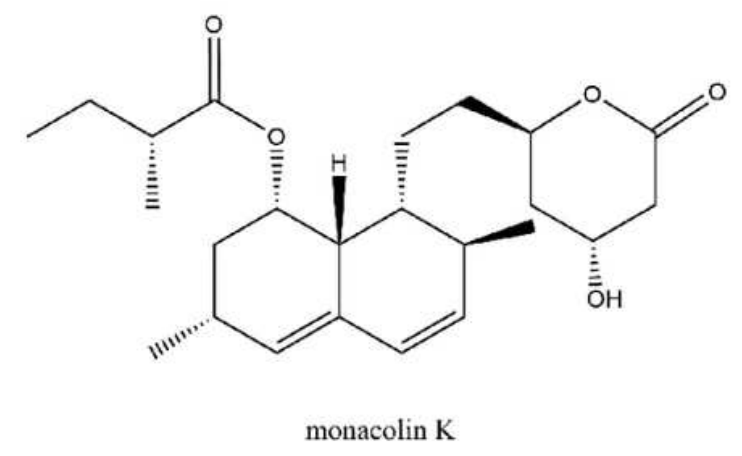

B Regoin 25.1 from B12

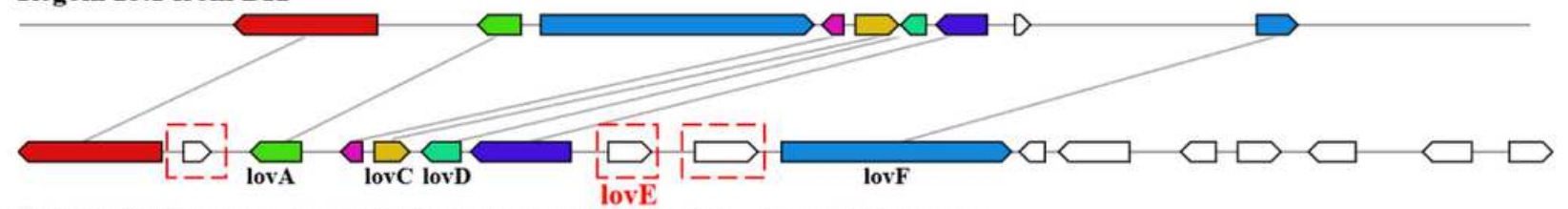

BGC0000088:monacolin K biosynthetic gene cluster from Aspergillus terreus

\begin{tabular}{lllll}
\hline Identifiers in Aspergillus terreus & Gene code & Product & Query cover & Identity \\
\hline AAD34550.1 & --- & esterase & 95 & 90 \\
AAD34551.1 & --- & unknown & -- & --- \\
AAD34552.1 & lovA & cytochrome P450 monooxygenase & 104 & 96 \\
AAD34553.1 & --- & unknown & 99 & 94 \\
AAD34554.1 & lovC & enoyl reductase & 114 & 86 \\
AAD34555.1 & lovD & transesterase & 100 & 99 \\
AAD34556.1 & --- & hydroxymethylglutaryl-coenzyme A reductase & 81 & 96 \\
AAD34557.1 & lovE & regulatory protein & -- & -- \\
AAD34558.1 & --- & unknown & -- & -- \\
AAD34559.1 & lovF & polyketide synthase & 100 & 96 \\
\hline
\end{tabular}

Figure 8

Putative monacolin K BGC in B12. A.The structure of monacolin K. B.The homologous genes of region 25.1 in B12 and terrein BGC known in Aspergillus terreus have been marked with the same color, and the missing genes are marked with red dotted boxes. Known BGCs description and amino acid homology (query cover and identity) were listed in the table. 


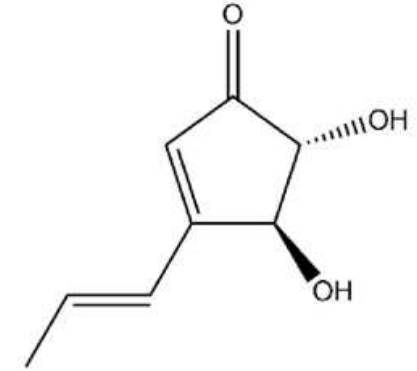

Terrein (1)

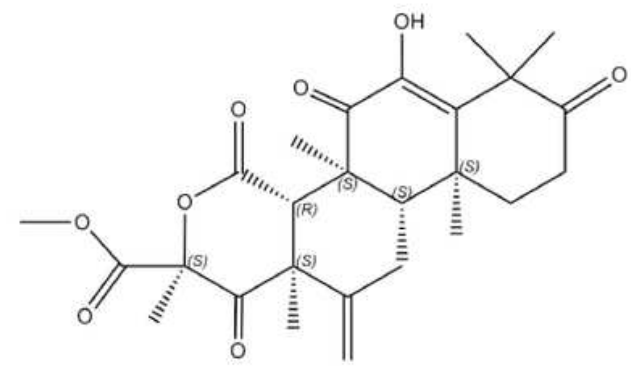

Terretonin A (4)

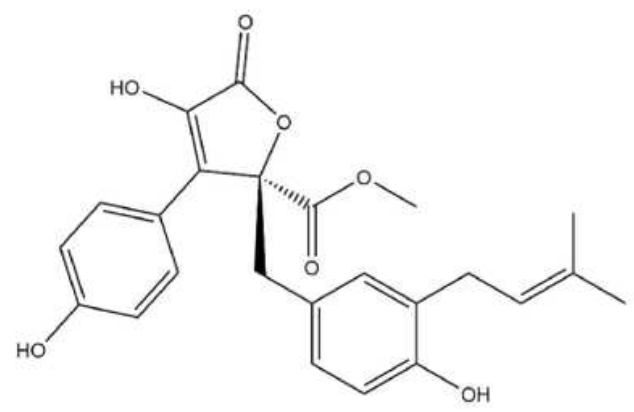

Butyrolactone I (2)

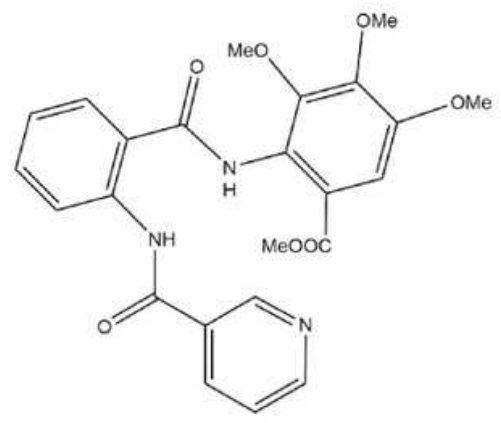

Acoapetaline B (5)

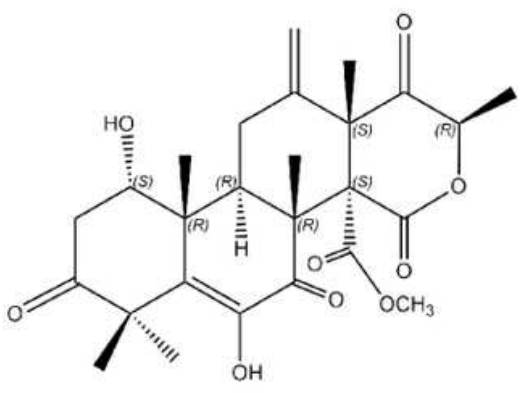

Terretonin E (3)

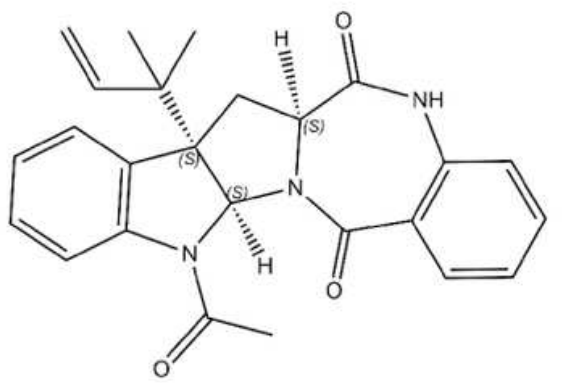

epi-Aszonalenin A (6)

Figure 9

The compounds was isolated from B12 
A

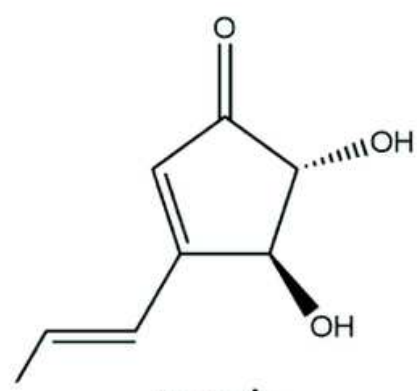

terrein

B

Regoin 9.4 from B12

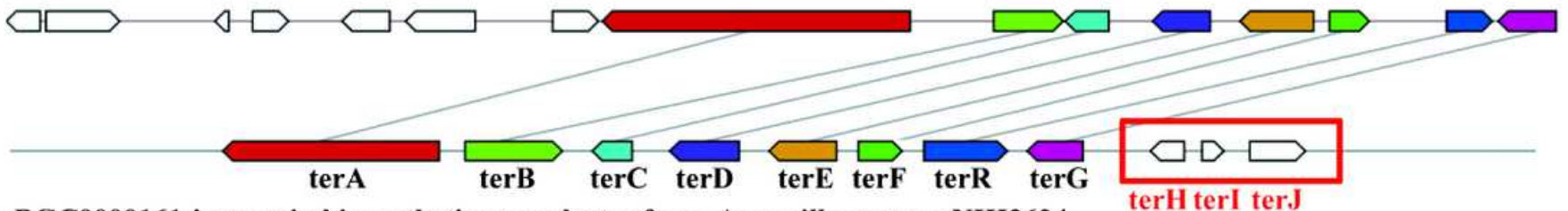

BGC0000161:isoterrein biosynthetic gene cluster from Aspergillus terreus NIH2624

\begin{tabular}{|c|c|c|c|c|}
\hline Identifiers in Aspergillus terreus NIH2624 & Gene code & Description & Query cover & Identity \\
\hline ATEG_00135 & terJ & major facility transporter (MFS) & --- & --- \\
\hline ATEG_00136 & terI & $\begin{array}{l}\text { protein of the superfamily of bleomycin resisitance } \\
\text { protein, glyoxalase I, and type I ring-cleaving } \\
\text { dioxygenanses }\end{array}$ & --- & --- \\
\hline ATEG_00137 & terH & NAD dependent epimerase/dehydratase & --- & --- \\
\hline ATEG_00138 & terG & major facility transporter (MFS) & $100 \%$ & $100 \%$ \\
\hline ATEG_00139 & terR & $\mathrm{Zn}_{2} \mathrm{Cys}_{6}$ transcriptional regulator & $99 \%$ & $72 \%$ \\
\hline ATEG_00140 & terF & protein with kelch motif & $123 \%$ & $80 \%$ \\
\hline ATEG_00141 & terE & multicopper oxidase & $94 \%$ & $91 \%$ \\
\hline ATEG_00142 & terD & FAD-dependent monooxygenase & $99 \%$ & $99 \%$ \\
\hline ATEG_00143 & terC & FAD-dependent monooxygenase & $144 \%$ & $68 \%$ \\
\hline ATEG_00144 & terB & $\begin{array}{l}\text { DH-KR, multidomain protein with dehydratase and } \\
\text { ketoreductase function }\end{array}$ & $74 \%$ & $73 \%$ \\
\hline ATEG_00145 & terA & non-reducing polyketide synthase (NR-PKS) & $84 \%$ & $97 \%$ \\
\hline
\end{tabular}

\section{Figure 10}

Putative Terrein BGC in B12. A.The structure of Terrein. B.The homologous genes of region 25.1 in B12 and terrein BGC known in Aspergillus terreus have been marked with the same color, and the missing genes are marked with red dotted boxes. B.Known BGCs description and amino acid homology (query cover and identity) were listed in the table. 